\title{
Is a continuous rational social aggregation impossible on continuum spaces?
}

\author{
Wu-Hsiung U. Huang
}

Received: 12 February 2006 / Accepted: 15 September 2008 / Published online: 4 November 2008 (C) Springer-Verlag 2008

\begin{abstract}
This paper provides a global topological setting for the social choice theory on continuum spaces of alternatives, in contrast to the local differentiable setting of Chichilnisky. Chichilnisky proved that a rational continuous social choice must be discontinuous in her setting. Our paper revisits her theorem to trace the source of this discontinuity. We find that the discontinuity is irrelevant to social aggregation, per se. The main theorem states that there exist a number of continuous social utility maps which are anonymous and satisfy the Pareto condition. As a corollary, we show that there exist corresponding continuous social welfare functions, if singularity is not separated from regular preferences in social preference topology. This extends the possibility result of Jonnes-Zhang-Simpson on linear preferences, to the general ones. The notion of singularity of preferences, relative to the given mathematical structure of an alternative space, is carefully studied.
\end{abstract}

\section{Introduction}

Following Arrow's nonexistence theorem (Arrow 1951) of a rational social choice for the finite discrete case of alternatives, Chichilnisky set up a local differentiable framework on continuum alternative spaces and analogously proved (Chichilnisky 1980, 1982) that there exists no continuous social welfare function which is anonymous and respects unanimity. Baigent (1987) applied Chichilnisky's continuity back to the finite discrete case to derive his notion of proximity preservation, and, like Chichilnisky, showed its inconsistency with unanimity and anonymity. In Huang (2004), the author of this paper analyzed the notion of proximity preservation both on the discrete and continuum cases, finite or infinite, and questioned its rationality by proving the rigidity.

W.-H. U. Huang ( $\varangle)$

Department of Mathematics, National Taiwan University, Taipei 106, Taiwan

e-mail: yshhng@yahoo.com; whuang@math.ntu.edu.tw 
Jones et al. (2003) considered linear preferences by revising Chichilnisky's topology for social preferences to allow null preference. In this particular case, they obtained a possibility result that there exists continuous, anonymous social aggregations respecting unanimity. But a possibility result about linear preferences does not generally support the possibility of a continuous rational social aggregation. To show the impossibility, it is logically sound to restrict oneself to linear preferences, as in Chichilnisky $(1980,1982)$. However, to claim the possibility, we need to consider general preferences, since linear preferences constitute only a very small portion of specific preferences (with measure zero). Besides, the meaning of the revised topology allowing null social preference in the given sense requires further study.

We first examine local differentiable settings of the continuum framework, which was first considered by Antonelli (Debreu 1972) and developed by Chichilnisky to derive her impossibility theorem. We examine certain weakness in the model and remark that singularities such as saturation points or plainly indifference sets are not consistently defined. In contrast to the local differentiable model, we establish a global topological setting that allows us to study singularities in a systematic way. In this setting it is shown that the impossibility of a continuous rational social aggregation is not a problem of social aggregation itself. In fact, we prove an existence theorem of a continuous "social utility map",

$$
U: P^{N} \longrightarrow C^{o}(X),
$$

which is anonymous and satisfies the strong Pareto condition-and thus respects unanimity (see Theorem A in Sect. 1.4 and Theorem 3 in Sect. 3.2). Here, utility space $C^{o}(X)$ of continuous functions defined on $X$ is equipped with the most common and natural topology for $C^{o}(X)$, i.e., the compact-open topology, which corresponds to the uniform convergence on compact sets. Preference space $P$ is equipped with a canonical topology $\Im$, which is essentially analogous to Chichlnisky's topology in the local differentiable setting. Further comments on the result are as follows:

(I) Based on the existence of social utility map $U$, it is evident that the nonexistence of a continuous rational social welfare function is caused by the discontinuity of map $\pi$ from $C^{o}(X)$ to preference space $P$. Therefore the nonexistence theorem of Chichilnisky is irrelevant to social aggregation itself. In other words, if we decompose her social welfare function into $\pi \circ U$, i.e.,

$$
F: P^{N} \stackrel{U}{\longrightarrow} C^{o}(X) \stackrel{\pi}{\longrightarrow} P,
$$

the discontinuity does not occur on the first map, i.e., social aggregation $U$. It occurs on the second map $\pi$, which is simply the mathematical quotient map obtained by discarding the cardinality of social utility functions while preserving the ordinality for social preferences.

The above analysis raises two questions:

(i) Is a continuous "rational" social utility map $U: P^{N} \rightarrow C^{o}(X)$ less desirable than a continuous "rational" social welfare function $F: P^{N} \rightarrow P$ ? Which one 
is more satisfactory from the point of view of social aggregation itself? As a corollary of the existence of $U$, we prove that there exists a continuous rational social choice map in a weak sense (see Corollary $\mathrm{C}$ of Sect. 1.5).

(ii) In reality, does the social preference space carry a topology same as that of individual preference space? Given that social aggregation apparatus can be much more accurate and sophisticated, should we consider some other topology for social preference space? We will try the zero order topology $\mathfrak{\Im}_{O}$ (see Definition 2.5.1) as a possible topological model for social preference space. By a quotient theorem (Huang 1996), $\mathfrak{\Im}_{O}$ is equivalent to $C^{o}(X) / \sim$ (The latter was also denoted by $F / \sim$ in Chichilnisky's notation (Chichilnisky 1980), yet not well studied). Theorem B (of Sect. 1.5) shows the existence of infinitely many social welfare functions

$$
F: P^{N} \longrightarrow\left(P, \Im_{o}\right)
$$

which are unanimous, anonymous and continuous relative to social preference topology $\mathfrak{\Im}_{o}$.It is interesting to note that the revised topology, defined by Jones-Zhang-Simpson for linear preferences, is basically the subtopology of the zero order topology $\mathfrak{\Im}_{o}$. Their related possibility result about linear preferences is extended to general preferences in Theorem $\mathrm{B}$ of this paper.

(II) Generally speaking, a topology on a set of objects means a consistent way to define how the objects vary continuously, or equivalently, to define how a sequence of the objects converges. That is why topology is a significant mathematical tool to describe various natural and social phenomena. In this paper, we not only consider the given topology on alternative space $X$, but also introduce a suitable topology for preference space $P$, the totality of preferences, in order to study how preferences vary continuously, based on the given topology of $X$.

A preference considered in this paper is a (continuous) preference order globally defined on $X$ (See Sect. 1.3 for precise definition). This definition is conventional and has been given by Mas-Collel et al. (1995). It can be regarded as equivalent (by Proposition 2.3.2) to a decomposition of alternative set $X$ into (closed) indifference sets $I_{\alpha}$ with a linear order. We call such a decomposition a stratification of $X$. Accordingly, an adequate topology that we introduce to preference space $P$ should respect the stratification structure.(For precise definition, see Sect. 2.3.)

In other words, a topology for $P$ is adequate if it is defined by the following criterion:

Criterion A A sequence of preferences $p_{n}$ of $P$ converges in the topology to a preference $p_{o} \in P$, if and only if the following two conditions are satisfied:

(A1) For any two indifferent sets $I_{\alpha}$ and $I_{\beta}$ of $p_{o}$ (not of $p_{n}$ ), it holds that $I_{\alpha} \succ I_{\beta}$ in $p_{o}$ iff $I_{\alpha} \succ I_{\beta}$ in $p_{n}$, for all large $n$. (This is equivalent to say that: given $x$ and $y$ in $X, x \succ y$ in $p_{o}$ iff $x \succ y$ in $p_{n}$ for all large $n$.)

(A2) The indifference sets of $p_{n}$ approximate to those of $p_{o}$ uniformly on compact sets. (The precise meaning of approximation is given in Theorem 1 of Sect. 3.)

The stratification topology $\mathfrak{I}$ introduced in this paper is defined on the basis of Criterion A. (It can be regarded as the first order topology of $P$, in contrast to the zero 
order topology $\mathfrak{\Im}_{o}$.) But Chichilnisky's topology $\mathfrak{\Im}_{c}{ }^{1}$ does not meet this criterion-see Example 1 to 4 in Sect. 1.2.

We will define the concept of "singularity" ("topological singularity" for precision) in the global topological setting. A preference $p$ in $P$, on which topology $\Im$ is equipped, is call singular, if $p$ has an indifference set which is not thin, i.e., the indifference set contains an open subset of alternative space $X$. Otherwise, $p$ is called regular.

Contrast to our setting, Chichilnisky's setting is local and differentiable, and hence the concept of "singularity" in her setting is relative to the differentiable structure of $X$. It means that a preference in her setting is regular if all the indifference sets are differentiable hypersurfaces of $X$. Otherwise, it is singular.

The difference between the two topologies $\mathfrak{\Im}$ and $\mathfrak{\Im}_{c}$ is illustrated particularly by the fact that $\mathfrak{I}_{c}$ maintains the separation property which states that

(a) a sequence of singular preferences can never tend to a regular preference, and

(b) a sequence of regular preferences can never tend to a singular one,

while $\Im$ satisfies only the semi-separation property, which is defined just by (b). Note that the semi-separation property is a direct result of condition (A2).

(III) The prime difficulty in obtaining a continuous rational social welfare function is that the semi-separation property holds for topology $\mathfrak{I}_{c}$ and $\mathfrak{s}$, when either of the two topologies is imposed upon the social preference space. For example, inputting a sequence of individual profiles into a social aggregation apparatus, let the outcome be a sequence of social utility functions:

$$
f_{n}(x)=\frac{x}{n}, \quad x \in[0,1] \equiv X,
$$

Evidently, $f_{n}(x)$ converges to function $f_{o}(x)=0$. The semi-separation property forces preference $p_{n}$, which corresponds to utility function $f_{n}\left(i . e ., \pi\left(f_{n}\right)=p_{n}\right)$, staying away from(and therefore not tending to) preference $p_{o}$, which corresponds to $f_{o}\left(\right.$ i.e., $\left.\pi\left(f_{o}\right)=p_{o}\right)$, since $p_{n}$ is regular and yet $p_{o}$ is singular. In other words, $p_{n}$ does not converge to $p_{o}$, although $f_{n}$ converges to $f_{o}$. This is the essential reason for discontinuity of map $\pi$. And it makes continuity of a rational social welfare function impossible.

However, it is not the chosen topology $\mathfrak{\Im}_{c}$ or $\mathfrak{\Im}$ that should be blamed, because at least the semi-separation property(b) is a logical consequence of acceptable condition (A2) of Criterion A. The discrepancy between continuity of the cardinality-discarding function $\pi$ and the semi-separation property of the preference topology is innate.

Now there are two reasonable stances to be held while viewing the discrepancy. First, we observe that the prime difficulty occurs only on map $\pi$, which projects the utility functions to the corresponding preferences, after social aggregation has been accomplished. So the discontinuity of social welfare functions has nothing to do with social aggregation itself. In this light, Theorem A in Sect. 1.4, as well as Corollary C

\footnotetext{
1 Chichilnisky's original topology is not well-defined, when singularities are included. Topology $\mathfrak{\Im}_{c}$ (see Sect. 1.1 for precise definition) is a modified form based on her original topology. For the analysis, see Remark A in Sect. 1.1 and Remark B in Sect. 1.3 of his paper.
} 
in Sect. 1.5, support the stance and clarify the long confusion about the impossibility of continuous rational social choice.

The second stance is to discard the semi-separation property, and define another topology for the social preference space by requiring only (A1) of Criterion A, which we call the zero-order topology and denote by $\mathfrak{\Im}_{o}$. It is then not difficult to see the existence of a required continuous social welfare function $F: P^{N} \rightarrow\left(P, \Im_{o}\right)$, which is stated in Theorem B of Sect. 1.5. However, is $\mathfrak{\Im}_{O}$ a topology more acceptable for the social preference space than $\mathfrak{s}$ (and than $\mathfrak{\Im}_{c}$ )? In Examples 8 and 10, we give certain practical evidence to support the second stance. Nevertheless, the question remains open for further investigation.

(IV) The idea of proving Theorem A is based on the global topological setting of our model of preference space $P$. Chichilnisky's framework is a local differentiable setting in the sense that a preference is defined by directions of differentiable local preferences. Instead, preference space $P$ adopted in this paper is the space of continuous "preference orders" (also called weak orders), defined globally on alternative space $X$.

The global topological setting may fit reality more closely, at least in a conventional way. It happens to have an advantage that the inferior set $Q_{x}^{\prime}(p)$ of a preference $p$ (see Sect. 2.1 for definition) to a given alternative $x$ in $X$, is consistently defined and, therefore, can be measured, so that the lifting of an individual preference $p$ to a utility function $f$ is possible (see Theorem 2). Thus the average rule can be selected as a candidate for the desired social utility map $U$.

However, given $x$ in $X$, inferior set $Q_{x}^{\prime}(p)$ is not continuous in $p$. Technically, this is the major problem that we have to solve for showing the existence of utility map $U$. It makes the main arguments of this paper nontrivial even mathematically.

Nevertheless, the measure ${ }^{2}$ of inferior set $Q_{x}^{\prime}(p)$ is lower semi-continuous in $p$, i.e.,

$$
\liminf \mu\left(Q_{x}^{\prime}\left(p_{n}\right)\right) \geq \mu\left(Q_{x}^{\prime}\left(p_{o}\right)\right)
$$

as $p_{n}$ tends to $p_{o}$ in $\mathfrak{\Im}$, where "lim inf" denotes the limit infimum and $\mu(*)$ denotes the measure. Consider the singular set $\Lambda(p)$ of $p$, which is defined by the union of all the indifference sets containing interior singular points of $p$ (see Definition 2.4.2). We prove that

$$
\mu\left(Q_{x}^{\prime}(p)-\Lambda(p)\right)
$$

is continuous in $p$. It means that a modification with the term $\Lambda(p)$ makes the measure $\mu\left(Q_{x}^{\prime}(p-\Lambda(p))\right.$ continuous in $p$. By letting

$$
f(x)=\mu\left(Q_{x}^{\prime}(p)-\Lambda(p)\right)+1>0
$$

we may lift each individual preference $p_{i}$ to a utility function $f_{i}$ continuously (see Theorem 2). Then applying the average rule, for example, by

$$
U\left(p_{1}, \ldots, p_{N}\right)=\left(f_{1}+\cdots+f_{N}\right) / N
$$

\footnotetext{
2 The notion of measure of a set may be regarded as a generalized concept of the "area" of a set in a plane. See Definition 2.6.6.
} 
the desired social utility map $U$ is obtained. Theorems A, the main result, as well as Theorem B and Corollary C stated at the end of Sect. 1 are then proved.

(V) To investigate the possibility of a rational social aggregation, it seems that singular preferences should not be precluded from consideration. Chichilnisky's impossibility is the extension of Condorcet paradox to the continuum framework. However, the impossibility occurs because of the preclusion of singularities. Singularities deserve to be carefully treated, as they always exist in reality, though complicated technically. In our model, we study systematically the role of singularity, noting the compatibility between the notion of singularity and the concerned structure of the alternative space (Sect. 2.6). Based on the study of singularities, we establish the lifting theorem by adjusting the term of the inferior set $Q_{x}^{\prime}(p)$ to $Q_{x}^{\prime}(p)-\Lambda(p)$, i.e., subtracting from $Q_{x}^{\prime}(p)$ singular set $\Lambda(p)$, and thus finding continuous social utility maps with certain principles of rationality. This makes the existence of the required continuous social utility map possible.

Since the setting and the proofs given in this paper involve many technical concepts of topology, we illustrate the ideas and main results of the paper with many examples and figures in Sect. 1. In fact, Sect. 1 is provided for general readers, who are not particularly familiar with mathematical language of topology. We include Table $\Omega$ at the end of Sect. 1 to summarize the contents.

Section 2 is devoted to rigorous formulation of the framework, pointing out the difficulties involved. In Sect. 3, we prove the theorems, again presenting examples to explain certain subtle points. Since parts of the arguments are too technical to be included in the text, we leave them for Appendix.

Throughout this paper, one may assume alternative space $X$ a closed n-cube $\mathbf{I}^{n}$ of real n-space $\mathbf{R}^{n}$, a closed or open ball in $\mathbf{R}^{n}$, or a quadrant $\mathbf{R}_{+}^{n}=\left\{\left(x_{1}, x_{2}, \ldots, x_{n}\right) ; x_{i} \geq\right.$ $0, \forall i\}$. However, the assumption is made only for easier understanding. ${ }^{3}$ In fact, all the theorems are valid for topological manifolds with or without boundary(partial or total), except that when $X$ is non-compact, certain standard modifications of the statements of the theorems are required. For instance, when $X$ is quadrant $\mathbf{R}_{+}^{n}$, the Jordan measure $\mu$ is infinite, but we may convert it into a finite one by taking arctan $r$ as the converting density function, so that Theorem 2 of Sect. 3.2 is still true. By a topological manifold, ${ }^{4}$ we mean a topological space, every point of which has an Euclidean-like neighborhood. An n-cube (closed or open), a quadrant in $\mathbf{R}^{n}$, any domain in $\mathbf{R}^{n}$, an $\mathrm{n}$-torus, and an n-sphere are the examples.

The case of infinite discrete alternatives has been considered in a joint work of Chen with this author (Chen and Huang 2008). The case is noteworthy, because it incorporates both the continuum case and the finite discrete case. The totality $P$ of preferences on an infinite discrete alternative space $X$ has non-discrete topology, although $X$ itself is discrete. Stratification topology $\mathfrak{\Im}$ of $P$ as well as the zero order topology $\mathfrak{\Im}_{O}$ introduced in this paper, would make the corresponding forms easier to be understood in that case. As $X$ itself is discrete, one may avoid technical topological formulation on $X$

\footnotetext{
3 Most of the theorems are valid for general topological spaces with mild conditions such as Tychonoff $T_{3}$, local-compactness, locally-connectedness, and connectedness.

${ }^{4}$ Precisely, a topological manifold $X$ is a topological space in which each point $x \in X$ has a neighborhood homeomorphic (i.e., topological equivalent) to the Euclidean space $\mathbf{R}^{n}$.
} 
and still somehow get the idea intuitively. However, the forms defining the topologies of $P$ for the case are derived from the theory established in this paper.

The author would like to express his gratitude to the referee and H.J.Chen for having brought the works of previous contributors to his attention.

\section{Background and results}

\subsection{A sketch of Chichilnisky's theorem}

Assume that alternative space $X$ has a differentiable structure. Following Chichilnisky's formulation (Chichilnisky 1982), one consider a local differentiable setting $S_{c}=\left(P_{c}, \mathfrak{\Im}_{c}\right)$, which consists of preference space $P_{c}$ on $X$ and topology $\mathfrak{\Im}_{c}$ imposed on $P_{c}$. A preference in $P_{c}$ is defined by an unit vector field $v$ differentiable on $X$, except $v$ may be zero at some points of $X .^{5}$ The points where $v$ is zero are called singular points of $v$, and the rest are called regular. ${ }^{6}$ A metric $d_{c}$ is introduced to $P_{c}$ by

$$
d_{c}(v, w)=\sup _{x \in X}|v(x)-w(x)|, \quad \forall v, w \in P_{c} .
$$

Metric $d_{c}$ induces a topology $\Im_{c}$ on $P_{c}{ }^{7}$

Chichilnisky proved that there exists no continuous function

$$
F: P_{c}^{N} \longrightarrow P_{c}
$$

which is anonymous $(\mathrm{AN})^{8}$ and respects unanimity (UN), ${ }^{9}$ where $P_{c}^{N}$ denotes the product space of $N$ copies of $P_{c}$ and $N$ denotes the number of individuals involved in social aggregation $F$. Her proof is elegant and simple. For easier understanding of our later content, let us sketch the idea in a few lines. Let $X=\mathbf{R}_{+}^{n}$ or $I^{n}$, and let $L \subset P_{c}$ be the totality of linear preferences in $P_{c},{ }^{10}$ i.e.,

$$
L \equiv\left\{v \in P_{c}: v \text { is either the zero field or constant in } x \text { with }|v(x)|=1, \forall x \in X\right\} .
$$

\footnotetext{
5 Chichilnisky considered only two cases that $v$ may be zero on the whole $X$ or on a set of measure zero. As we will study singularity systematically here, we do not consider this restrain.

6 Note that $v$ represents the most desirable direction which is perpendicular to the differentiable indifference hypersurfaces of the preference. Also note that $v$ is zero at a point of $X$, if and only if around that point the indifference set is not a differentiable hypersurface.

7 When the singular sets of preference vectors are non-empty, there is a slight difference between Chichilnisky's formulation and the above setting $S_{c}$. The latter can be regarded as a completion of the former. For details, see Remark $\mathrm{A}$ at the end of this subsection.

8 (AN): $F\left(p_{\sigma_{1}}, p_{\sigma_{2}}, \ldots, p_{\sigma_{N}}\right)=F\left(p_{1}, p_{2}, \ldots, p_{N}\right), \forall\left(p_{1}, p_{2}, \ldots, p_{N}\right) \in P_{c}^{N}$ and $\forall$ permutation $\sigma$ of the indices $1,2, \ldots, N$.

9 (UN): $F(p, p, \ldots, p)=p, \forall p \in P_{c}$.

10 For $X$ a general manifold, we may choose a point $x_{o}$, with a coordinate neighborhood $U$, regarded as a part of $R^{n}$. Consider a constant vector field $v$ in $U$ which is of unit length or zero. Denote $v\left(x_{o}\right)$ by $v_{o}$. Let $v$ be zero outside $U$. Call such a vector field a linear preference relative to $x_{o}$ and denote its totality by $L \subset P_{C}$. Then the proof also follows.
} 
It is clear that $L$ is topologically equivalent ${ }^{11}$ to $\mathbf{S}^{n-1} \cup\{\mathbf{0}\}$, where $\mathbf{S}^{n-1}$ is the $(n-1)$-dimensional unit sphere in $\mathbf{R}^{n}$ and $\mathbf{0}$ means the zero vector of $\mathbf{R}^{n}$. Suppose that $F$ is continuous. Then $F$ restricted to $L^{N} \subset P_{c}^{N}$, should also be continuous, where $L^{N}=L \times L \times \cdots \times L$ (of $N$ copies). We illustrate the proof by considering the case that $N=2$ and $n=2$, depicting the idea in a sketch to show how the impossibility occurs. For general $N$ and $n$, it needs more sophisticated mathematical argument.

Select an interior point $x_{o} \in X$. Consider the localization map $\psi: P_{c} \longrightarrow \mathbf{S}^{1} \cup\{0\}$ defined by

$$
\psi(v)=v\left(x_{o}\right) \in \mathbf{S}^{1} \cup\{\mathbf{0}\}
$$

Obviously, $\psi$ is continuous. This is seen by the definition of $d_{c}$ which provides uniform convergence of preference vector fields on $X$. Let $F^{\prime}=\left.F\right|_{L^{2}}$ be the map of $F$ restricted to its subset $L^{2}$ of $P_{c}^{2}$. We have the composition map

$$
F^{\prime \prime}=\psi \circ F^{\prime}: L^{2} \stackrel{F^{\prime}}{\longrightarrow} P_{c} \stackrel{\psi}{\longrightarrow} \mathbf{S}^{1} \cup\{\mathbf{0}\},
$$

which is continuous, since both $F^{\prime}$ and $\psi$ are continuous. Now $L^{2}$ is topologically equivalent to $\left(\mathbf{S}^{1} \cup\{\boldsymbol{0}\}\right)^{2}$ which contains four components. Among them there is a torus $\mathbf{S}^{1} \times \mathbf{S}^{1}$. Since the image of a connected set under a continuous map must be connected, $F^{\prime \prime}$ either maps $\mathbf{S}^{1} \times \mathbf{S}^{1}$ into $\mathbf{S}^{1}$ or into $\{\mathbf{0}\}$, i.e.

$$
F^{\prime \prime}: \mathbf{S}^{1} \times \mathbf{S}^{1} \longrightarrow \mathbf{S}^{1} \text {, or } F^{\prime \prime}: \mathbf{S}^{1} \times \mathbf{S}^{1} \longrightarrow\{\mathbf{0}\},
$$

But the unanimity of $F$ precludes the latter case. ${ }^{12}$ It then suffices to consider $F^{\prime \prime}$ : $\mathbf{S}^{1} \times \mathbf{S}^{1} \longrightarrow \mathbf{S}^{1}$. Clearly, $F^{\prime \prime}$ is still anonymous and respects unanimity in its mathematical form. Let $F^{\prime \prime}$ be interpreted by the following figure :

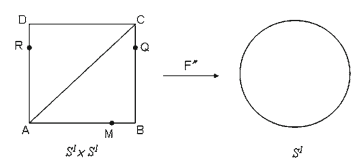

Fig. 1

Here segment $A B$ is pasted on $C D$, and $A C$ on $B D$. By this, rectangle $A B D C$ is regarded topologically the torus $\mathbf{S}^{1} \times \mathbf{S}^{1}$. By anonymity, $F^{\prime \prime}(M)=F^{\prime \prime}(R)$, for any two points $M \in A B$ and $R \in A C$ which are symmetric with respect to axis $A D$. (Also, $F^{\prime \prime}(R)=F^{\prime \prime}(Q)$, since $A C$ is pasted on $B D$.) Hence the map of $F^{\prime \prime}$ on $A B$ duplicates the map $F^{\prime \prime}$ on $B D$. Therefore $F^{\prime \prime}$ on $A B \cup B D$ must cover range $\mathbf{S}^{1}$ an even number of times. However, by unanimity, $F^{\prime \prime}$ on diagonal $A D$ covers range $\mathbf{S}^{1}$ exactly once. Thus the two maps $F^{\prime \prime}$ on $A D$ and $F^{\prime \prime}$ on $A B \cup B D$ can not be homotopic to each other.

\footnotetext{
11 Two topological spaces $X$ and $Y$ are topologically equivalent, if there exists one to one correspondence $f: X \longrightarrow Y$ such that both $f$ and $f^{-1}$ are continuous. By $f$ continuous, we mean $\forall A$ open in $Y, f^{-1}(A)$ is open in $X$. A topological equivalence is also called a homeomorphism.

12 Any element $v_{o}$ in $S^{1} \cup\{0\}$ has a $v \in L_{2}$ such that $v\left(x_{o}\right)=v_{o}$. By unanimity of $F$, we have $F(v, v, \ldots, v)=v$. Therefore, $F^{\prime}\left(v_{o}, \ldots, v_{o}\right)=\Psi \circ F(v, \ldots, v)=\Psi(v)=v\left(x_{o}\right)=v_{o}$.
} 
This contradicts the fact that the former can be deformed along triangle $\triangle A B D$, to the latter, as $F^{\prime \prime}$ is continuous on $\mathbf{S}^{1} \times \mathbf{S}^{1}$. Therefore (AN), (UN) and the continuity of $F$ are logically inconsistent.

Remark A Chichilnisky considered (Chichilnisky 1980, 1982) various subspaces $P_{c}^{*}$ of $P_{c}$ with topology $\mathfrak{\Im}_{c}^{*}$ slightly different from $\mathfrak{\Im}_{c}$. Here we explain why in place of her setting $S_{c}^{*}=\left(P_{c}^{*}, \mathfrak{\Im}_{c}^{*}\right)$, we have to take $S_{c}=\left(P_{c}, \mathfrak{\Im}_{c}\right)$ as the model representing various local differentiable settings to be compared with our global topological setting $S=(P, \mathfrak{I})$. First we list the various forms of her setting as follows.

Form $\alpha: P_{c}^{*}=$ the space of $C^{r}$ codimension-1 transversely oriented foliations of choice space $X=$ the space of locally integrable $C^{r}$ vector fields of $X$ which is nowhere zero. The corresponding topology $\mathfrak{\Im}_{c}^{*}$ is given by $d_{c}$ which is defined in (†).

Form $\beta: P_{c}^{*}=$ the locally integrable $C^{r}$-vector fields of $X$ whose singular set may be the whole space $X$ or have measure zero. She defined topology $\mathfrak{\Im}_{c}^{*}$ by

$$
\begin{gathered}
p_{j} \rightarrow p \text { iff } \\
\sup _{x \in X-\cup_{1}^{\infty} S_{j}}\left|p^{j}(x)-p(x)\right| \rightarrow 0,
\end{gathered}
$$

(Chichilnisky 1982, pp 349), i.e., $\Im_{c}^{*}$ is given by " $d_{c}$ outside the singular set" as she claimed.

Form $\gamma: P_{c}^{*}=F / \sim$, where $F$ is the space of $C^{r+1}$ real valued functions on $X$ and $\sim$ is the obvious equivalent relation. Topology $\mathfrak{\Im}_{c}^{*}$ is inherited from the $C^{r+1}$ sup norm of $F$.

Form $\delta: P_{c}^{*}=$ the subspace of all convex preferences of $F / \sim$ in form $\gamma . \Im_{c}^{*}$ is the corresponding subspace topology.

Setting $S_{c}=\left(P_{c}, \Im_{c}\right)$ is introduced to modify $S_{c}^{*}=\left(P_{c}^{*}, \mathfrak{\Im}_{c}^{*}\right)$ and provides a unified form of local differentiable settings. When singularities are precluded, $\Im_{c}^{*}$ of form $\alpha$ is the same as $\mathfrak{\Im}_{c}$. However, to include singularities, setting $S_{c}^{*}$ seems invalid, and that is why we have to replace it with $S_{c}$. Here are the comments about $S_{c}^{*}$ :

(1) $P_{c}^{*}$ of form $\beta$ allow only special kinds of singularities to be considered. That means singularities can not be studied systematically. Furthermore, $\mathfrak{\Im}_{c}^{*}$ of form $\beta$ is not a well-defined topology. There exists a sequence $p_{j}$ which converges to $p$ in $\mathfrak{\Im}_{c}^{*}$, but has subsequences not convergent to $p$ in $\mathfrak{\Im}_{c}^{*}$. For instance, there exists a sequence $\left\{p_{1}, p_{2}, p_{3}, \ldots, p_{n}, \ldots\right\}$ convergent to $p$ in $\Im_{c}^{*}$, but $\left\{p_{2}, p_{3}, \ldots, p_{n}, \ldots\right\}$ is divergent in $\mathfrak{\Im}_{c}^{*}$. This makes the notion of continuity not consistently defined.

In fact, given a well-defined topology, defined by a family of open sets $\left\{U_{\alpha} ; \alpha \in\right.$ an index set $I\}$, sequence $p_{j}$ converges to $p$, if and only if for any $U_{\alpha}$ containing $p$, there exists $N$ such that $p_{j} \in U_{\alpha}$, for all $j>N$. As a consequence, any subsequence of a sequence $p_{j}$ convergent to $p$ must converge. However, $\Im_{c}^{*}$ fails to satisfy this subsequence convergence property(See Appendix (AIVa) for proof). 
(2) $\Im_{c}^{*}$ fails to satisfy SCP, the last property, partly because the corresponding sup norm,

$$
d_{c}^{*}(p, q)=\sup _{x \in X-S_{q}}|p(x)-q(x)|,
$$

using which Chichilnisky wrote down formula $(* *)$, does not define a distance. Clearly, $d_{c}^{*}$ is not symmetric in $(p, q)$.

(3) If $d_{c}^{*}(p, q)$ is adjusted by a symmetric form, $\sup _{x \in X-S_{p} \cup S_{q}}|p(x)-q(x)|$, it still does not define a distance, since the triangular inequality does not hold (For proof, see Appendix (AIVb)). Therefore Chichilnisky's idea of defining a topology which intends to ignore singularities is not realized. This is why a modified distance $d_{c}$ has to be defined by the above formula ( $\dagger$ ) to replace $d_{c}^{*}$. Correspondingly, topology $\mathfrak{\Im}_{c}$ defined by $d_{c}$ is to replace topo logy $\mathfrak{\Im}_{c}^{*}$ defined by $d_{c}^{*}$.

(4) The sup norm formula (**) implies that a singular point of $p$ must be a singular point of $p_{n}$ for some $n$, if $p_{n}$ converges to $p$ in $\mathfrak{\Im}_{c}^{*}$.This is unacceptable as well, because convergence concerns only eventual behavior of the sequence.

(5) Topology $\Im_{c}^{*}$ of form $\gamma$ and $\delta$ are invalid as well. If these forms are considered, Chichilnisky's proof of her impossibility theorem will not be valid. (See Appendix (AIVc) for explanation.)

\subsection{The preference convergence}

The local differentiable setting $S_{c}=\left(P_{c}, \Im_{c}\right)$ will be examined when comparing it with our global topological setting $S=(P, \mathfrak{I})$. First we illustrate that many good convergent preferences are divergent in $S_{c}$.

Example 1 Consider a sequence $\left\{p_{n}: n=1,2, \ldots\right\}$ in $P_{c}$ and $p_{o} \in P_{c}$ with their indifference curves as in Fig. 2 , where arrows $v_{n}$ and $v_{o}$ denote their preference vector fields respectively. Here $v_{n}$ and $v_{o}$ are indicated by unit vector fields, perpendicular to the indifference curves with the directions illustrated, except they are zero at $O_{n}$ and $O$ respectively. Let $O_{n} \rightarrow O$, as $n \rightarrow \infty$. Practically, sequence $p_{n}$ is regarded convergent to $p_{o}$. As $p_{n}$ and $p_{o}$ satisfy Criterion $\mathrm{A}, p_{n}$ converges to $p_{o}$ in our stratification topology $\mathfrak{s}$.

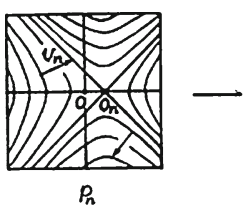

$P_{n}$

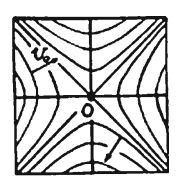

P。

Fig. 2

However, $p_{n}$ does not converge to $p_{o}$ in local differentiable topology $\mathfrak{\Im}_{c}$, since

$$
d_{c}\left(p_{n}, p_{o}\right)=\sup _{x \in X}\left|v_{n}(x)-v_{o}(x)\right|=\left|v_{n}(O)-v_{o}(O)\right|=1
$$


for any $n$. Note that $v_{n}(O)=$ a unit vector pointing to the right, and $v_{o}(O)=$ the zero vector, hence the last equality is 1 . (If we use Chichilnisky's formula $(*)$, we also have

$$
d_{c}^{*}\left(p_{n}, p_{o}\right)=\sup _{x \in X-\cup S_{n}}\left|v_{n}(x)-v_{o}(x)\right|=\left|v_{n}(O)-v_{o}(O)\right|=1,
$$

where the singularity set $S_{n}$ of $p_{n}$ is $\left\{O_{n}\right\}$. Note that $O \in X^{\prime}=X-\cup S_{n}$ and hence $O$ is counted in the last formula. Thus the sup norm does not tend to zero and $p_{n}$ does not converge to $p_{o}$.)

Example 2 Let $X=[-1,1] \times[-1,1]$. Consider preferences $p_{n}$ and $p_{o}$ on $X$ as described in Fig. 3, where the indifference sets of $p_{n}$ and $p_{o}$ are given by the concentric circles with center $(0,0)$ and by the dark areas

$$
\left\{(x, y) \in I^{2} ; x^{2}+y^{2} \leq\left(a+\frac{1}{n}\right)^{2}\right\} \text { and }\left\{(x, y) \in I^{2} ; x^{2}+y^{2} \leq a^{2}\right\}
$$

of $p_{n}$ and $p_{o}$, respectively. Note that $a \geq 0$ is a given real number. When $p_{n}$ and $p_{o}$ are regarded as preferences in $P_{c}$, preference vectors $v_{n}$ and $v_{o}$ are either all outward along radial directions, or all zero on the dark areas.

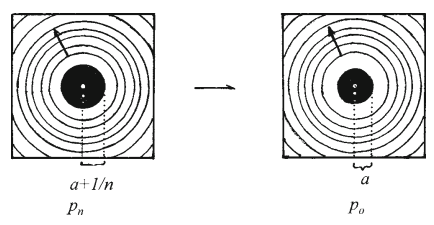

Fig. 3

In our topology $\mathfrak{I}, p_{n}$ converges to $p_{o}$, as Criterion $\mathrm{A}$ is satisfied. But it diverges in $\mathfrak{\Im}_{c}$. (As for $S_{c}^{*}=\left(P_{c}^{*}, \mathfrak{\Im}_{c}^{*}\right.$ ), where forms $\alpha$ and $\beta$ are the possible models, $p_{n}$ and $p_{o}$ are not even considered as preferences, since they have singular sets that are neither the whole space $X$ nor of measure zero.)

Example 3 Let $X=[0,1] \times[0,1] \subset \mathbf{R}^{2}$, or let $X$ be the infinite first quadrant in the plane. Consider preferences $p_{n}$ in Fig. 4 such that: (1) the indifference sets are exactly the hyperbolas,

$$
\left\{(x, y) \in X ;(x-\alpha)(y-\alpha)=\frac{1}{n}, x-\alpha \geq 0 \text { and } y-\alpha \geq 0\right\},
$$

Namely, for each fixed $n$, the hyperbolas may be obtained by translating $x y=\frac{1}{n}$ along vectors $(\alpha, \alpha)$, for any real number $\alpha$.

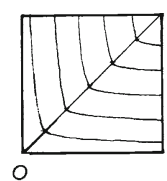

$p_{n}$

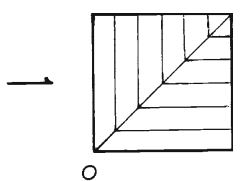

$p_{o}$

Fig. 4 
(2) the corresponding preference vectors $v_{n}$ point away from the origin $(0,0)$.

Define $p_{o}$ similar to $p_{n}$, except the indifference hyperbolas having their equations replaced with $(x-\alpha)(y-\alpha)=0$. Again $p_{n}$ converges to $p_{o}$ in our topology $\Im$, since they satisfy Criterion A, but not in setting $S_{c}$. (Also, $p_{n}$ does not converge to $p_{o}$ in setting $S_{c}^{*}$, since the singular set of $p_{n}$ is empty, and the sup norm in formula (**) is attained, for any $n$, by diagonal points $x^{\prime}$ of $X$, where $\left|v_{n}\left(x^{\prime}\right)-v_{o}\left(x^{\prime}\right)\right|=1$.)

Definition Given a continuous function $f$ on $X \rightarrow \mathbf{R}$, which may be regarded as a utility function on $X$, we say a preference $p$ on $X$ corresponds to $f$, when

$$
x \succsim y \quad \text { in } \quad p \text { iff } f(x) \geq f(y), \forall x, y \in X .
$$

We also say that $p$ is defined by utility function $f$.

Example 4 Consider $X=[0,1] \subset \mathbf{R}$ for easier illustration. Suppose $f$ is a smooth function on $[0,1]$. Let $n$ be a positive integer. Divide $[0,1]$ into $2 n$ intervals of equal length. Define $f_{n}$ by a continuous function which satisfies

$$
f_{n}(x)=f\left(\frac{k}{n}\right), \quad \text { for } x \in\left[\frac{k}{n}, \frac{k}{n}+\frac{1}{2 n}\right] \quad \text { and } \quad k=0,1,2, \ldots, n-1 \text {, }
$$

making it linear on the remaining subintervals as in Fig. 5.

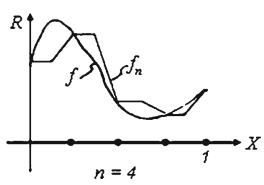

Fig. 5

Let $p_{n}, p$ be the preferences defined by utility functions $f_{n}, f$, respectively. Then $f_{n}$ converges uniformly to $f$. As $p_{n}$ and $p$ satisfy Criterion A, we have $p_{n}$ convergent to $p$ in $\mathfrak{\Im}$, but divergent in $\mathfrak{\Im}_{c}$. (In setting $S_{c}^{*}, p_{n}$ is not included in preference space $P_{c}^{*}$.)

The inadequacy of $S_{c}$ shown by the above examples is due to topology $\mathfrak{\Im}_{c}$ given by $d_{c}(v, w)=\sup \{|v(x)-w(x)| ; x \in X\}$, which forces two preferences far apart (to a distance of least 1) from each other, as long as the singular sets of $v$ and $w$ are not completely identical.

For instance, in Example 1, origin $O=(0,0)$ is a singular point of $p_{o}$, but it is regular for $p_{n}$. According to $\mathfrak{\Im}_{c}$ or any of $\mathfrak{\Im}_{c}^{*}, p_{n}$ does not converge to $p_{o}$, although singular point $O_{n}$ tends to $O$. In Example 2, the singular set, i.e., the dark area in the figures of $p_{n}$, deforms evenly into the singular set of $p_{o}$ as $n \rightarrow \infty$, but in $\Im_{c}, p_{n}$ does not converge to $p_{o}$. This occurs because in order to have $p_{n}$ convergent to $p_{o}$, $\Im_{c}$ requires that $\forall n>$ some $n_{o}$, the singular set of each $p_{n}$ should be the same as that of $p_{o}$. The same happens in Examples 3 and 4. The strict requirement makes local differential topology $\mathfrak{\Im}_{c}$ "too strong" (i.e., having too many open sets) to contain considerable cases of convergence. 


\subsection{Globalization of vector fields}

A continuous preference order (also called a global preference hereafter) on a continuum alternative space $\mathrm{X}$ is normally defined by a complete transitive binary relation $\succsim{ }^{13}$ on $\mathrm{X}$ such that for each $x$ in $\mathrm{X}$, the weak preferred set $\{y \in \mathrm{X} ; y \succsim x\}$ and the weak inferior set $\{y \in X ; y \precsim x\}$ are closed in $X$. Preference space $P$ considered in this paper is the totality of such continuous preference orders.

Debreu (1972) has analyzed the cases where a local preference may be integrated into a global preference order, i.e., a vector field $v$ in $P_{c}$ may be integrated to obtain a preference order in $P$ by a trajectory argument. More precisely, when $v$ satisfies some integrability condition, ${ }^{14}$ we first define $x \sim y$, iff $x$ and $y$ are in the same indifference set as given by $v$, or in mathematical terminology, iff $x$ and $y$ are on the same integral manifold with tangent spaces perpendicular to $v$ at each point of the manifold. One may then use the trajectory argument to define the preference order basically by stating that $x \succsim y$ if there exists a trajectory curve from $y$ to a point $z$ with $z \sim x$.

However, even when the required integrability condition is satisfied, there may be no adequate preference order in $P$, corresponding to $v$ in $P_{c}$.

Example 5 Define a vector field $v \in P_{c}$ on $\mathrm{X}=[-1,1] \times[0,1]$ by

$$
v\left(x_{1}, x_{2}\right)= \begin{cases}(-1,0), & x_{1}<0 \\ (1,0), & x_{1}>0 \\ (0,0), & x_{1}=0\end{cases}
$$

then for any two alternatives $x=\left(x_{1}, x_{2}\right)$ and $y=\left(y_{1}, y_{2}\right)$ with $x_{1} y_{1}<0$, we can not determine whether $x \precsim y$ or $x \succsim y$, although $v$ has been clearly defined. Thus the completeness condition is not satisfied.
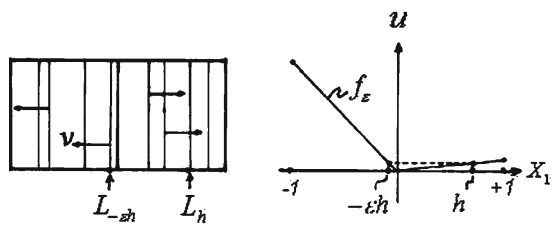

Fig. 6

Surely one may assign $x \precsim y$ or $x \succsim y$ to the pair $x, y$ in a consistent way, e.g., let $f_{1}$ be defined by $f_{1}\left(x_{1}, x_{2}\right)=\left|x_{1}\right|, \forall\left(x_{1}, x_{2}\right) \in X$. Then preference order $p_{1}$, "defined by" utility function $f_{1}$ (as in the definition before Example 4), is consistent with vector field $v$. However, there are infinitely many preference orders corresponding

\footnotetext{
13 By a binary relation on $\mathrm{X}$, we mean a subset $G \subset \mathrm{X} \times \mathrm{X}$. This induces $\succsim$ defined by $x \succsim y$ iff $(x, y) \in G$. We call $\succsim$ complete, if $\forall x, y \in \mathrm{X}, x \succsim y$ or $y \succsim x$ or both. If $x \succsim y$ and $y \succsim x$, we say $x$ and $y$ are indifferent, and denote it by $x \sim y$. We also call $\succsim$ transitive, if $\forall x, y, z \in \mathrm{X}, x \succsim y$ and $y \succsim z \Rightarrow x \succsim z$. A binary relation which is complete and transitive is usually called a weak order or a rational preference relation (see Mas-Collel et al. 1995).

14 Such as Frobenius integrability condition (see any introductory text book on differential geometry, e.g., S.Sternberg: Lectures on Differential Geometry, Prentice-Hall, p132); for further analysis see Debreu Debreu (1972).
} 
to the same vector field $v$. For instance, let $p_{\epsilon}$ be the preference order defined by utility function $f_{\epsilon}$ which is given by

$$
f_{\epsilon}\left(x_{1}, x_{2}\right)= \begin{cases}-x_{1}, & x_{1} \leq 0 \\ \epsilon x_{1} & x_{1}>0\end{cases}
$$

where $\epsilon$ is a small positive number. Let $L_{h}$ denote the vertical straight line segment $\left\{\left(h, x_{2}\right) ; x_{2} \in[0,1]\right\}$. Given each $h \in(0,1], L_{-\epsilon h} \cup L_{h}$ is an indifference set of $p_{\epsilon}$ (see Fig. 6). Thus we have many different preference orders $p_{\epsilon} \in P$ corresponding to the same $v \in P_{c}$, which leads to certain ambiguity.

Example 6 Let $X=I^{2} \subset \mathbf{R}^{2}$ and $v \in P_{c}$ be the unit vector fields pointing counterclockwise along the concentric circles with center $(0,0)$, except at $(0,0)$ it is zero. Let $x=\left(\frac{1}{2}, 0\right), y=\left(-\frac{1}{2}, 0\right)$. Then according to the trajectory argument, $x \succ y$ and $y \succ x$ occur simultaneously. Hence there is no preference order corresponding to $v$.

Remark $B$ As we have noticed in Remark A of Sect. 1.1, Chichilnisky attempted to define "globally integrable foliations" by considering $F / \sim$. But once singularities are included, the related topology makes $P_{c}^{*}$ a non-Hausdorff space and her theorem fails. Thus the attempt to consider global preferences is unsuccessful, and her formulation remains local when singularities are included in preference space.

\subsection{The global topological setting}

We now compare $\mathfrak{s}$ and $\mathfrak{\Im}_{c}$. As the topological structure is the focus of our concern, we define in the following the notion of singularity for preferences in $P$ relative to the topological structure of $X$, rather than relative to its differentiable structure.

A topological structure of $X$ can not detect differentiability of level sets. For instance, in Example 3, the cusps of level sets of $p_{o}$ appeared at the diagonal points of $[0,1] \times[0.1]$ are not detectable. Thus the cusps are not regarded singular relative to the topological structure of $X$.

On the other hand, the saddle points in Example 1 are not abnormal compared to other points, unless a manifold structure has been introduced to $X$. In this sense, saddle points are not regarded as singular points relative to a given topological structure.

However, whether a point is an interior point of the level set is detectable. It is natural to define singularity relative to topological structure as follows. Given $p \in P$, a point $x \in \mathrm{X}$ is called an interior singular point of $p$ if $x$ has an open neighborhood contained in indifference set $I_{x}(p) \equiv\{y \in \mathrm{X} ; y \sim x$ in $p\}$. If there exists such a point $x$ in $X$, preference $p$ is called (topologically) singular. Otherwise, $p$ is regular.

Let $\Lambda(p)$ be the union of the indifference sets each of which contains an interior singular point. We call each point of $\Lambda(p)$ a singular point of $p$ and the set $\Lambda(p)$ the singular set of $p$. For example, preferences $p_{n}$ and $p_{o}$ (when $a>0$ ), as described in Examples 2 and 4, are singular, with their singular sets denoted by the dark circular disks in Fig. 3 and at least the odd subintervals in Fig. 5, while those in Examples 1 and 3 are regular.

Comparing $\mathfrak{I}$ with local differentiable settings which include Chichilnisky's framework, one can see a difference. If an indifference set has an interior singular point, 
the preference direction at that point must be zero. Therefore, an interior singular point in our global topological setting is also singular in a local differentiable setting, but not vice versa. In fact, in a local differentiable setting, $O_{n}$ for $p_{n}$ and $O$ for $p_{o}$ in Example 1 are singular points, which make $p_{n}$ and $p_{o}$ singular, but they are regular in our global topological framework.

As stated in (II) of Introduction, we consider Criterion $\mathrm{A}$ as the criterion of convergent preferences. Based on Criterion A, stratification topology $\mathfrak{I}$ is defined (see Definition 2.5.2 for precise definition).

Now we introduce the notion of the separation property to study $\mathfrak{\Im}$ and $\mathfrak{I}_{c}$ as follows.

For $p_{n}$ to be convergent to $p_{o}$ in $\mathfrak{\Im}_{c}$, it is required that the singular set of $p_{o}$ must be identical with that of $p_{n}$ for all large $n$. Thus a sequence of singular preferences never converge to a regular(i.e., non-singular) preference in $\Im_{c}$. Yet it is not so in $\Im$, since we allow the (topological)singular sets of $p_{n}$ deforming from their boundaries gradually to become those of $p_{o}$. In Example 2 where $a=0$, or in Example 4, we have seen a sequence of singular preferences converging to a regular preference, if we adopt $\mathfrak{s}$.

Namely, the following separation property holds in $\Im_{c}$, but not in $\Im_{\text {. }}$

Separation property: The set $\Sigma$ of singular preferences and the set $\Theta$ of regular preferences are separated ${ }^{15}$ from each other, i.e., there exist two disjoint open sets $\Phi$ and $\Psi$ in the preference space, with $\Sigma \subset \Phi$ and $\Theta \subset \Psi$, or equivalently, a sequence of singular preferences never converges to a regular preference, and vice versa.

Example 7 Let $u$ and $u_{n}: I^{2}=[0,1] \times[0,1] \rightarrow \mathbf{R}$ be defined by $u_{o}(x, y)=f(x)$, $u_{n}(x, y)=f_{n}(x)$, where $f$ and $f_{n}$ are given in Example 4 and it is assumed that $f$ is strictly monotone. Denote by $p_{o}$ and $p_{n}$ the preferences on $I^{2}$, defined by utility functions $u_{o}$ and $u_{n}$ respectively. Then $p_{o}$ is regular, while $p_{n}$ is singular and $p_{n}$ converges to $p_{o}$ in $\mathfrak{s}$, but not in $\mathfrak{\Im}_{c}$. The dark stripes in Fig. 7 are the singular sets $\Lambda\left(p_{n}\right)$. We have $p_{o} \in \Theta$ and $p_{n} \in \Sigma$. The convergence shows that there is no open set $\Psi$ containing $p_{o}$ in $\Theta$, such that $\Psi$ is disjointed from the set $\Sigma$ of singular preferences.

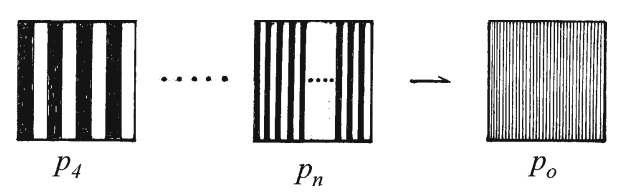

Fig. 7

This example illustrates that the separation property does not hold in $\mathfrak{s}$, although it does in $\mathfrak{I}_{c}$.

However, both $\mathfrak{s}$ and $\mathfrak{\Im}_{c}$ adopt the following semi-separation property.

Semi-separation Property A sequence of regular preferences never converges to a singular preference, i.e., $\exists \Phi$, open in the preference space, such that $\Sigma \subset \Phi$ and $\Phi \cap \Theta$ are empty.

\footnotetext{
15 Two sets $A$ and $B$ in a topological space $\Omega$ are called separated from each other, if there exist two disjoint open sets $U$ and $V$ such that $A \subset U$ and $B \subset V$.
} 
As an example, a sequence of linear preferences never converge in both $\mathfrak{\Im}_{c}$ and $\mathfrak{s}$ to the null preference $o$.

Definition The null preference $o$ is the preference with indifference set $I_{x}(o)=$ $X, \forall x \in X$.

Remark Given two non-empty disjoint subsets $A$ and $B$ of a general topological space $\Omega$ such that $A \cup B=\Omega$. If $A$ and $B$ are separated from each other, then $\Omega$ is (at least) disconnected. But the semi-separation property defined by the existence of an open set $U \supset A$ such that $U \cap B=\phi$, may keep $\Omega$ still connected. This general topological fact is seen by letting $\Omega=[-1,1], A=(0,1]$ and $B=[-1,0]$, and choosing $U=A$. Note that $1 / n \in A$, and $0 \in B$, but $1 / n$ converges to 0 , where $n=1,2,3, \ldots$. Considering that $A$ represents the set $\Sigma$ of singular preferences and $B$ represents the set $\Theta$ of regular ones, we see that $\Sigma \cup \Theta$ may still be connected. However if we also require $\Sigma$ to be closed in the preference space, then $\Sigma \cup \Theta$ is disconnected, since $\Sigma$ now is open and closed in $\Sigma \cup \Theta$.

The semi-separation property is compatible with reality, when preference space $P$ is the totality of individual preferences. This is because individuals are insensitive to very slight change of their preferences for distinct alternatives from indifference $(\sim)$ to strict preference $(\succ)$. We construct an example to explain this observation.

Example 8 Consider an individual who in the beginning has his initial preference $p_{o}$ indifferent on all alternatives of $X=I^{2}$, i.e., $p_{o}=o$, the null preference. After a while, his preference begins to change very slightly on the alternatives, for example, taking a linear preference $p_{\epsilon}$ which is defined by a linear utility function $f_{\epsilon}$ with extremely small gradient $\left|\nabla f_{\epsilon}\right|=\epsilon>0$. If at this moment, he was asked to declare precisely his preference in front of an apparatus of social aggregation, e.g., he was asked to vote, it can hardly be imagined that he would claim $p_{\epsilon}$ rather than $p_{o}$, since he is not so sensitive, when $\epsilon$ is arbitrarily small, say, $\epsilon=0.0000 \ldots 01$. (Note that no matter how small a positive number $\epsilon$ is, $p_{\epsilon}$ is the same as $p_{1}$ such that the preferences on any two alternatives with different $x$-coordinates are different.) Namely, $p_{\epsilon}$ should not be regarded by him as a gradual change of $p_{o}$. This is why $\mathfrak{I}_{c}$ and $\mathfrak{\Im}$ do not accept $p_{\epsilon}$ as convergent to $p_{o}$ for individual preferences. The indifference sets of $p_{\epsilon}$ and $p_{o}$ are illustrated in Fig. 8.
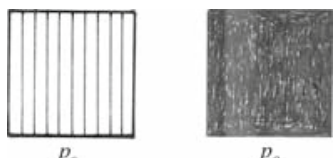

$p_{o}$

Fig. 8

Using $\Im$ as the preference topology on $P$, we will prove our main theorem in Sect. 3.

Theorem A There exist infinitely many continuous social utility maps,

$$
U: P^{N} \rightarrow C^{o}(X),
$$


which are anonymous and satisfy the strong Pareto condition ${ }^{16}$ (and hence respect unanimity. ${ }^{17}$ )

\subsection{The discontinuity}

Theorem A shows the existence of continuous rational social utility maps. In fact, it shows the existence of a rational continuous social aggregation which provides social utilities distributed on the given set $\mathrm{X}$ of alternatives. Why our result seems contradictory to the discontinuity theorem of Chichilnisky?

(1) The discontinuity of the social welfare function proved by Chichilnisky arises from the separation property applied to social preference spaces by $\mathfrak{\Im}_{c}$. Recall her work sketched in Sect. 1.1. The separation property makes the range space of

$$
F^{\prime \prime}: \mathbf{S}^{1} \times \mathbf{S}^{1} \longrightarrow \mathbf{S}^{1} \cup\{\mathbf{0}\}
$$

a disconnected set $\mathbf{S}^{1} \cup\{0\}$, where $\mathbf{S}^{1}$ represents the totality of linear preferences (which are regular) and $\{0\}$ represents the null preference (which is singular). The contradiction is then derived from the disconnectedness of $\mathbf{S}^{1} \cup\{\mathbf{0}\}$. And the impossibility theorem is therefore established.

In other words, the essential reason why discontinuity occurs in Chichilnisky's theorem is that the possible singularities of social preference are precluded from the regular ones. The preclusion is based on the semi-separation property.

Generally speaking, social aggregation may naturally lead to mutual cancellation among individual preferences with various preference directions. For example, linear preferences of opposite directions, or of Condorcet triple, cancel out each other. The cancellation would result in singularity on many occasions. Namely, the resultant preference would possibly become degenerate and null, for which no particular direction is preferred. Thus it becomes singular. However, the semi-separation property of topology $\mathfrak{\Im}_{c}$ forces the singularity to remain separated from regular preferences. If social aggregation cancels the opposite preferences gradually, and tends to a singularity with all directions indifferent, then discontinuity is automatically the outcome.

That is, any topology of social preference space admitting the semi-separation property will automatically induce discontinuity in a social aggregation. This is what Chichilnisky's theorem really says.

(2) Nevertheless, if we consider the composition of maps

$$
F: P^{N} \stackrel{U}{\longrightarrow} C^{o}(X) \stackrel{\pi}{\longrightarrow} P
$$

\footnotetext{
16 By $U$ satisfying the strong Pareto condition, we mean $\pi \circ U$ satisfies the strong Pareto condition, or equivalently, if $x \succsim y$ in $p_{i}, \forall i$, then $f(x) \geq f(y)$ where $f \equiv U(\bar{p})$; also if $x \succ y$ in $p_{i}, \forall i$, then $f(x)>f(y)$. Here $\pi$ is the projection map given in (I) of Introduction.

17 By $U$ respecting unanimity, we mean $\pi \circ U$ respects unanimity.
} 
we have a social welfare function $F$, where $F=\pi \circ U$ and $\pi$ is the projection map from the utility function space to the preference space, i.e., $\pi(f) \in P$ is the preference defined by the given utility function $f \in C^{o}(X)$. Obviously, it is map $U$, not map $\pi$, which involves social aggregation. In our topological global setting, recall we have the advantage, depicted in (IV) of Introduction, that $Q_{x}^{\prime}(p)$ can be defined and measured. With this advantage, we use the continuity of the measure of $Q_{x}^{\prime}(p)-\Lambda(p)$ to prove Theorem A, which shows the existence of $U$ with required continuity and other rationality. Consequently, the nonexistence of $F$ is reduced to discontinuity of $\pi$.

However, discontinuity of $\pi$ is caused by the (semi-)separation property imposed on the topology chosen for social preference space $P$. Or more precisely, discontinuity of $\pi$ happens because of the incompatibility of the two topologies of utility function space $C^{O}(X)$ and social preference space $P$.

We explain the incompatibility by the following example.

Example 9 Consider $X=[0,1]$ and two sequences of utility functions

$$
f_{n}(x)=\left(\frac{1}{n}+1\right) x, \quad g_{n}(x)=\frac{x}{n} .
$$

Clearly, $f_{n}(x)$ tends to $f(x)=x$ and $g_{n}(x)$ tends to $g(x)=0$. However, the two sequences of preferences $p_{n}$ and $q_{n}$ defined by $f_{n}$ and $g_{n}$ respectively are unfortunately the same sequence of preferences, i.e.,

$$
p_{n}=\pi\left(f_{n}\right)=\pi\left(g_{n}\right)=q_{n},
$$

while preferences $p=\pi(f)$ and $q=\pi(g)$ defined respectively by their limits $f$ and $g$ of $f_{n}$ and $g_{n}$ are distinct.

In fact, $\forall x, y \in[0,1]$,

$$
x \prec y \text { in } p, \quad \text { iff } x<y .
$$

but $x \sim y$ in $q$. Thus $p \neq q$. Clearly, $p$ is regular but $q$ is singular. Now we face the dilemma, whether we should define sequence $p_{n}\left(=q_{n}\right)$ as convergent to $p$, or define it as convergent to $q$ ? Practically, only one case can be chosen. In both topologies $\mathfrak{s}_{c}$ and $\mathfrak{\Im}$, where the semi-separation property holds, it is required that

$$
\lim p_{n}\left(=\lim q_{n}\right)=p, \text { but not }=q,
$$

which says that

$$
q_{n}=\pi\left(g_{n}\right) \text { does not tends to } q=\pi(g),
$$

although $g_{n}$ tends to $g$.

(3) The above analysis might change the long accepted interpretation of Chichilnisky's impossibility theorem. Now we see that it is not a continuous rational social aggregation impossible. The impossibility reduces to discontinuity of map 
$\pi$, which is irrelevant to social aggregation. And the discontinuity is caused by the semi-separation property imposed on the topology of $P$.

But do we have a better choice of a topology for $P$, such that $\pi$ is continuous and hence a rational social welfare function $F$ is continuous?

Example 9 says that even this is impossible, unless we accept a very weak topology for the social preference space, which is different from the topology of the individual preference space. More precisely, the dilemma of selecting $p$ or $q$ as the limit of $p_{n}\left(=q_{n}\right)$ always exists, unless we accept our topology to permit the existence of multiple limits of a single sequence and discard the semi-separation property.

For instance, when we accept zero ordered topology $\Im_{o}$ for $P$ defined by (A1) of Criterion A (see detail in Sect. 2.5), the projection map

$$
\pi=C^{o}(X) \rightarrow\left(P, \mathfrak{\Im}_{o}\right)
$$

is continuous. The proof is based on a quotient theorem of Huang (1996), which was proved earlier by this author. It states that $\mathfrak{\Im}_{O}$ equipped on $P$ is exactly the quotient topology of $C^{o}(X)$ under $\pi$. So $\Im_{o}$ and the topology of $C^{o}(X)$ are now compatible $e^{18}$ with each other. Combining the quotient theorem and Theorem A, we immediately obtain:

Theorem B There exist infinitely many continuous social welfare functions

$$
F: P^{N} \stackrel{U}{\longrightarrow} C^{o}(X) \stackrel{\pi}{\longrightarrow}\left(P, \Im_{o}\right)
$$

which are anonymous and satisfy the strong Pareto condition.

(4) To accept a sequence of regular preferences convergent to a singular preference, such as $q_{n}$ convergent to $q$ in Example 9, is not purely based on compatibility of the two topologies chosen for utility space and preference space. It might be previously built in the given topology of alternative space $X$. Here is an example:

Example 10 In Example 5, let $p_{o}$ denote the preference obtained by substituting $\epsilon=0$ in the definition of $f_{\epsilon}$. Indifference sets of $p_{\epsilon}$ with $\epsilon>0$ are vertical straight lines, while the indifference set of $p_{o}$ at $(0,0)$ is the whole square $I_{+}=\left\{\left(x_{1}, x_{2}\right) \in \mathrm{X}\right.$, $0 \leq x_{1} \leq 1$ \}. Given $x, y$ in $I_{+}$, we have for $p_{\epsilon}$,

$$
\begin{aligned}
& x=\left(x_{1}, x_{2}\right) \sim x_{\epsilon}=\left(-\epsilon x_{1}, x_{2}\right) \\
& y=\left(y_{1}, y_{2}\right) \sim y_{\epsilon}=\left(-\epsilon y_{1}, y_{2}\right) .
\end{aligned}
$$

As $\epsilon$ is arbitrarily small, $x$ and $y$ are only very slightly different in $p_{\epsilon}$. Note that the notion of the "slightness" mentioned here is not defined by certain "assumed" utilities, but by the fact that $x_{\epsilon}$ tends to $x_{o}=\left(0, x_{2}\right), y_{\epsilon}$ tends to $y_{o}=\left(0, y_{2}\right)$ and $x_{o} \sim y_{o}$ in $p_{\epsilon}$, for any $\epsilon \geq 0$. The notion of the "slightness" has been defined precisely by the given topology of alternative space $X$ itself. Therefore when a topology introduced for

$\overline{18}$ This is because that $U$ is open in $\left(P, \Im_{o}\right)$ iff $\pi^{-1}(U)$ is open in $C^{o}(X)$. 
social preferences is sufficiently sophisticated, it is expected to accept convergence of $p_{\epsilon}$ to $p_{o}$. And hence the semi-separation property (which rejects the convergence of $p_{\epsilon}$ to $p_{o}$ ) should be discarded from the social preference topology.

Example 10 gives evidence to support $\mathfrak{\Im}_{O}$ as a possible model of social preference topology, since it simply reflects the topology of alternative space $X$. Nevertheless, topology $\mathfrak{\Im}_{O}$ might not be accepted as a good model, because there exist multiple limits for a single sequence and therefore $\mathfrak{\Im}_{o}$ is not even a Hausdorff space.

(5) We may now claim that a continuous rational social choice is possible if we accept singularity. To make it precise, we define a social choice map by

$$
C: P^{N} \rightarrow 2^{X}
$$

where $\forall$ profile $\bar{p}=\left(p_{1}, \ldots, p_{N}\right) \in P^{N}, C(\bar{p})$ is a closed subset of $X$, and $2^{X}$ is the power set of $X$, i.e., the family of all the subsets of $X$. We say $C$ is continuous if the given $\bar{p}_{n}$ converges to $\bar{p}$ in $P^{N}$, and $x_{n} \in C\left(\bar{p}_{n}\right)$, it holds that

$$
\lim _{n \rightarrow \infty} x_{n} \in C(\bar{p})
$$

Then we have:

Corollary C There exist infinitely many continuous social choice maps which are anonymous and respect unanimity.

The proof is evident in the virtue of Theorem A. Given a utility $f(x)$ on $X$, we define the maximal set by

$$
M(f) \equiv\{x \in X ; f(x) \geq f(y), \forall y \in X\} .
$$

Since $X$ is assumed compact, we see that $M(f)$ is a non-empty compact set in $X$. Then $C=M \circ U$ is evidently the required social choice map.

We use the following Table $\Omega$ to summarize the notions and results considered in this paper and compare the three topologies $\mathfrak{\Im}_{c}$, $\mathfrak{I}_{\text {and }} \mathfrak{\Im}_{o}$ in terms of them.

\section{The framework}

\subsection{Definition of preferences}

Let $X$ be a space of alternatives, which mathematically is a pure set, or more generally a topological space, noting that a pure set may also be regarded as a discrete topological space. A preference order $p$ on $X$ is a binary relation $\succsim$ defined on some pairs of elements of $X$ such that

(i) (Completeness); $\forall x, y$ in $X$, either $x \succsim y$ or $y \succsim x$, or both,

(ii) (Transitivity); $\forall x, y, z$ in $X, x \succsim y$ and $y \succsim z \Rightarrow x \succsim z$, where $x, y, z$ may be distinct or non-distinct.

The set of all preference orders on $X$ is denoted by $P_{o}$. 
Table $\Omega$ Notions and results

\begin{tabular}{|c|c|c|c|}
\hline Topology $\tau=\cdots \rightarrow$ & $\begin{array}{c}\text { Modified } \\
\text { Chichilnisky's } \\
\text { topology } \Im_{c}\end{array}$ & $\begin{array}{c}\text { Stratification } \\
\text { topology } \mathfrak{I}\end{array}$ & $\begin{array}{l}\text { Zero order } \\
\text { topology } \mathfrak{I}_{O}\end{array}$ \\
\hline Criterion A (see Sect. 0$)$ & $N$ & Y & $N$ \\
\hline A1 as Nec.Cond. & $Y^{\dagger}$ & & $Y^{\ddagger}$ \\
\hline A2 as Nec.Cond. & $Y^{*}$ & $Y$ & $N$ \\
\hline A1 as Suff.Cond. & & & $Y^{\ddagger}$ \\
\hline A2 as Suff.Cond. & $N$ & $Y$ & $N$ \\
\hline Semi-Separation Prop. & Y & Y & $N$ \\
\hline Separation Property & Y & $N$ & $N$ \\
\hline $\begin{array}{l}\text { Continuity of } U \\
\text { if } \tau \text { adopted as } \\
\text { individual preference } \\
\text { topology }\end{array}$ & $N$ & $\begin{array}{l}Y \\
\text { (Theorem A) }\end{array}$ & $N$ \\
\hline $\begin{array}{l}\text { Continuity of } \pi \text { and } F \\
\text { if } \tau \text { adopted as } \\
\text { social preference } \\
\text { topology }\end{array}$ & $N$ & $N$ & $\begin{array}{l}Y^{* *} \\
\text { (Theorem B) }\end{array}$ \\
\hline
\end{tabular}

$Y=$ Yes; $N=$ No; $\mathrm{A} 1$ and $\mathrm{A} 2$ are given in Criterion A of Sect. 0

$Y^{\dagger}=$ Yes, when A1 is restated for $\Im_{c}$ equivalently by "preference vectors $p_{n}$ tends to $p_{o}$ ateach point of $X$, in the sense of vector convergence in Euclidean space $R^{n}$ "

$Y^{*}=$ Yes, and moreover, a condition A2a (which is stronger than A2) holds, where A2a means: "the singular sets of $p_{n}$ and $p_{o}$ are all identical for large $n "$.

Nec. Cond. $=$ Necessary Condition of preference convergence $p_{n} \rightarrow p$ under topology $\tau$

Suff. Cond. $=$ Sufficient Condition of preference convergence $p_{n} \rightarrow p$ under topology $\tau$

$Y^{\ddagger}=$ Yes, and moreover, A1 is a necessary/sufficient condition of $p_{n} \rightarrow p$ under $\Im_{O}$

$Y^{* *}=$ Yes, when $\Im$ is still adopted as individual preference topology

Let $p \in P_{o}$ and $\succsim$ be the binary relation defined by $p$. If specification is necessary, we write " $x \succsim y$ in $p$ ", otherwise " $x \succsim y$ " is written for simplicity. By " $x$ is preferred to $y$ (in $p$ )", we mean $x \succsim y$ but not $y \succsim x$, and such a case is denoted by " $x \succ y$ ". On the other hand, when $x \succsim y$ and $y \succsim x, x$ is called indifferent to $y$ (in $p$ ), and is denoted by " $x \sim y^{\prime \prime}$. Given $U, V$ subsets of $X$, if $\forall x \in U, y \in V, x \succsim y$, then we write

$$
U \succsim V
$$

Also by $x \succsim V$, we mean $x \succsim y, \forall y \in V$.

It is evident that both of the binary relations $\succ$ and $\sim$ are also transitive. Furthermore, we see

$$
x>y \text { and } y \sim z \Rightarrow x \succ z
$$

and

$$
x \sim y \text { and } y \succ z \Rightarrow x \succ z .
$$

A preference order $p$ on $X$ can also be regarded as a subset $G$ of $X \times X$, where $(x, y) \in G$ iff $x \succsim y$, such that the corresponding conditions of completeness and transitivity are satisfied. The subset $G$ is called the graph corresponding to $p$. 
Definition 2.1.1 For a topological space $X$, a preference order $p$ respects the topology of $X$ if the corresponding graph $G$ is closed in $X \times X$ with product topology.

A preference order $p$ respecting the topology of $X$ is also called " $a$ continuous preference on $X$ " in economics terminology, ${ }^{19}$ which is defined by the equivalent condition that for two sequences $x_{n}, y_{n}$ with $x_{n} \rightarrow x, y_{n} \rightarrow y$ in $X, x_{n} \succsim y_{n}$ in $p$ implies $x \succsim y$ in $p$.

The totality of such preference orders respecting the topology of $X$ is called the continuous preference space on $X$, and denoted by $P(X)$. For a given $p \in P(X)$ and $x \in X$,we call

$$
R_{x}^{\prime}(p):=\{y \in X ; y \succ x \text { in } p\}
$$

by the preferred set of $p$ at $x$, and

$$
Q_{x}^{\prime}(p):=\{y \in X ; y \prec x \text { in } p\}
$$

by the inferior set of $p$ at $x$. Clearly, $R_{x}^{\prime}(p)$ and $Q_{x}^{\prime}(p)$ are open in $X$. Similarly,we call

$$
R_{x}(p):=\{y \in X ; y \succsim x \text { in } p\}
$$

by the weak preferred set of $p$ at $x$, and

$$
Q_{x}(p):=\{y \in X ; y \preceq x \text { in } p\}
$$

by the weak inferior set of $p$ at $x$, both of which are evidently closed in $X$. We also define the indifference set by

$$
I_{x}(p):=\{y \in X ; y \sim x \text { in } p\}=R_{x}(p) \cap Q_{x}(p),
$$

which is evidently closed in $X$. When there arises no confusion, we may simplify the notations by $R_{x}, Q_{x}, R_{x}^{\prime}, Q_{x}^{\prime}$ and $I_{x}$ respectively.

By the notation ":=" or " $\equiv$ ", we mean "denoting" or "being defined by". This convention will be repeatedly used in the paper. In addition, we introduce several topological notations. Given a set $A$ in $X$, the closure $\bar{A}$ of $A$ is defined by the minimal closed set containing $A$, while the interior Int $A$ of $A$ is the maximal open set contained in $A$.

We remark that $\overline{R_{x}^{\prime}} \subset R_{x}$ and $R_{x}^{\prime} \subset$ Int $R_{x}$, but it may happen that $\overline{R_{x}^{\prime}} \neq R_{x}$ or $R_{x}^{\prime} \neq$ Int $R_{x}$, unless $p$ is "regular" relative to the topological structure (see Sect. 1.4 for definition). The boundary $\partial A$ of $A$ is the difference $\bar{A}-$ Int $A$. It is clear that $\partial A$ consists of the points, of which each neighborhood intersects both $A$ and $X-A$. Therefore $\bar{A}=\partial A \cup \operatorname{Int} A$. Also notice that for a topological singular preference $p$ in $P(X)$, it is false that $\partial R_{x}=R_{x}-R_{x}^{\prime}$ at singular points $x \in \Lambda(p)$ ( see also Sect. 1.4). The singular sets play a significant role in our framework.

19 See e.g., [MC-W-G] Mas-Colell et al. "Microeconomic Theory" (1995), Oxford Univ. Press. 
The term"preference" is used as a general term to mean a preference order in $P_{o}$, or a continuous preference order in $P(X)$, if there arises no confusion.

\subsection{Graphs and continuity}

Consider a preference $p$ in $P(X)$ on $X$. The corresponding graph $G$ is closed in $X \times X$. Given a set $S$ in $X \times X$, let $S_{x}$ and $T_{x}$ denote the subsets

$$
\begin{aligned}
& S_{x} \equiv\{(x, y) \in S ; y \in X\} \\
& T_{x} \equiv\{(y, x) \in S ; y \in X\} .
\end{aligned}
$$

In general, if $S_{x}$ and $T_{x}$ are closed in $X, \forall x \in X$, the set $S$ is not necessarily closed in $X \times X$. However, for the graph $G$ corresponding to a preference order $p \in P_{o}$, we claim that if $R_{x}$ and $Q_{x}$ are closed in $X, \forall x \in X$, then $G$ is closed in $X \times X$. The claim would help us to ensure that we have used the full strength of the assumption of $p$ respecting the topology of $X$, whenever we apply to $p$ the condition that $R_{x}$ and $Q_{x}$ are closed in $X, \forall x \in X$. Namely, we do not need to check whether $G$ itself is closed in $X \times X$, which usually is complicate.

Proposition 2.2.1 Let $p \in P_{o}$. Then $p \in P(X)$ (i.e., $p$ is continuous) if and only if for each $x \in X, R_{x}$ and $Q_{x}$ are closed in $X$. In other words, the graph

$$
G \equiv\{(x, y) \in X \times X ; x \succsim y \text { in } p\}
$$

is closed in $X \times X$, if and only if

$$
R_{x} \equiv\{y \in X ; y \succsim x \text { in } p\}, Q_{x} \equiv\{y \in X ; y \preceq x \text { in } p\}
$$

are closed in $X, \forall x \in X$.

Proof The proof of the necessity is evident. We show the sufficiency in the following steps.

Step 1 For $K$ a compact set in $X$, we first claim $\exists x_{o} \in K$ such that $x_{o} \succsim K$. Suppose there exists no such $x_{o}$ in $K$, then given $x \in K$, we have $y_{x} \in K$ with $y_{x} \succ x$. By $R_{y_{x}}$ closed in $X, Q_{y_{x}}^{\prime} \equiv\left\{z \in X ; y_{x} \succ z\right\}$ is open in $X$. There exists an open neighborhood $U_{x}$ of $x$ such that $U_{x} \subset Q_{y_{x}}^{\prime}$, i.e.,

$$
y_{x} \succ U_{x} .
$$

Let $\left\{U_{x_{1}}, \ldots, U_{x_{N}}\right\}$ be a finite subcover of $\left\{U_{x} ; x \in K\right\}$. Let $y_{o} \in K$ be an element in $\left\{y_{x_{1}}, \ldots, y_{x_{N}}\right\}$ such that

$$
y_{o} \succsim y_{x_{i}}, \quad \forall i=1,2, \ldots, N
$$

Then $y_{o} \succ U_{x_{i}} \forall i=1,2, \ldots, N$. But $\left\{U_{x_{i}} ; i=1,2, \ldots, N\right\}$ covers $K$. We have $y_{o} \succ K$, contradicting to $y_{o} \in K$. 
Step 2 Let $G^{c}$ be the complement $X \times X-G$. We claim that $G^{c}$ is open in $X \times X$, i.e., given $(x, y) \in G^{c}, \exists$ neighborhoods $V, W$ of $x, y$ respectively, such that $V \times W \subset G^{c}$. First, we remark that $(x, y) \in G^{c}$ iff $x \prec y$ in $p$. Since $R_{y}$ is closed in $X, Q_{y}^{\prime}$ is open, there exists a neighborhood $U$ of $x$ such that $U \subset Q_{y}^{\prime}$. Let $V$ be a compact neighborhood $V$ of $x$ with $V \subset U .^{20}$ By Step $1, \exists z \in V$ such that

$$
z \succsim V
$$

On the other hand, $Q_{z}$ is closed in $X$. Hence $R_{z}^{\prime}$ is open. But $y \succ U, z \in V \subset U$, we have $y \succ z$, i.e., $y \in R_{z}^{\prime}$. There exists a neighborhood $W$ of $y$ such that $W \subset R_{z}^{\prime}$, i.e.,

$$
W \succ z
$$

The transitivity of $p$ implies that $W \succ V$, i.e.,

$$
V \times W \subset G^{c}
$$

The proof is completed.

Proposition 2.2.2 Given $p \in P(X)$ and two points $x, y$ in $X$ with $x \prec y$ in $p$, there exist neighborhoods $U, V$ of $x, y$ respectively such that

$$
U \prec V \text { in } p \text {. }
$$

As $X$ is locally compact, we may require $U$ and $V$ compact in the last statement.

Proof Since $(x, y) \in G^{c}$, there exists a neighborhood of $(x, y)$ in $X \times X$ which lies in $G^{c}$. By the definition of product topology of $X \times X$, the neighborhood contains $U$ $\times V$ where $U$ and $V$ are neighborhoods of $x$ and $y$ in $X$ respectively. Thus $U$ and $V$ are as required.

Proposition 2.2.3 Given $p \in P(X)$ and $x, y \in X$. Suppose $x_{n} \rightarrow x$ and $y_{n} \rightarrow y$ in $X$ with $x_{n} \succsim y_{n}$ in $p, \forall n$. Then $x \succsim y$ in $p$.

Proof It follows directly from the closeness of the graph $G$.

Proposition 2.2.4 Given $p \in P(X)$. Let $K$ be a compact set in $X$, then the sets defined by

$$
\begin{aligned}
R_{K}^{\prime}(p) & \equiv\{x \in X ; \quad K \prec x\} \\
Q_{K}^{\prime}(p) & \equiv\{x \in X ; \quad x \prec K\}
\end{aligned}
$$

are open in $X$.

20 The existence of such $V$ is due to the assumption that $X$ is locally compact, and of $T_{3}$. 
Remark The proposition 2.2.4 provides us a convenient technique that we shall use often: Given $K$ compact in $X, x \notin K$, there exists a neighborhood $V$ of $x$, such that $K \prec V$. Also, we may assume $V$ a compact neighborhood of $x$, as long as $X$ is locally compact.

Proof In Step 1 of Proposition 2.2.1, we have shown the existence of a "maximal" element $x_{o}$ of $K$, i.e., $x_{o} \succsim K$ in $p$. The set $R_{x_{o}}^{\prime}(p)$ is known open. Now $R_{K}^{\prime}(p)=$ $R_{x_{o}}^{\prime}(p)$. Hence $R_{K}^{\prime}(p)$ is open, as claimed. Similarly, $Q_{K}^{\prime}(p)$ is open.

\subsection{Stratification structure and Examples}

A complete binary relation $p$ defined on an alternative space $X$ is a relation between any pair $(x, y)$ in $X$ which is independent of the relation between the pairs other than $(x, y)$. But when the complete binary relation $p$ also satisfies the transitivity, $p$ stratifies the space into indifference sets with a linear order. Namely, a preference order on $X$ defines a stratification of $X$.

The converse is also true, i.e., a stratification of $X$ corresponds uniquely a preference order on $X$. The equivalence between a stratification and a preference order is valid, even when $X$ is a topological space. Of course, as the topology of $X$ is now concerned, the stratification structure should also respect the topology of $X$, in the sense that the indifference sets are closed in $X$. We define an abstract stratification structure as follows.

Definition 2.3.1 Given a topological alternative space $X$, a stratification $\sigma$ is a decomposition of $X$ into non-empty closed subsets $I_{\alpha}, \alpha \in A$, associated with an injective assignment of a non-empty subindex set $A_{\alpha}$ of $A$ to each $\alpha \in A$, where $A$ is an index set, such that

(S1) $X=\cup_{\alpha \in A} I_{\alpha}$, and $I_{\alpha} \cap I_{\beta}=\phi, \forall \alpha, \beta \in A$ with $\alpha \neq \beta$, (Decomposition)

(S2) $R_{\alpha}$ and $Q_{\alpha}$ are closed in $X$, where (Topological coherence)

$$
R_{\alpha} \equiv \cup_{\beta \in A_{\alpha}} I_{\beta}, \quad Q_{\alpha} \equiv\left(X-R_{\alpha}\right) \cup I_{\alpha}
$$

(S3) $A_{\alpha} \cap A_{\beta}=A_{\alpha}$ or $A_{\beta}$, (Linear ordering of inclusion)

(S4) $\alpha \in A_{\alpha}, \forall \alpha \in A ; A_{\beta} \subset A_{\alpha}$, whenever $\beta \in A_{\alpha}$. (Minimality of $\alpha$ in $A_{\alpha}$ )

We denote the stratification $\sigma$ by $\left(X, I_{\alpha} ; A, A_{\alpha}\right)$.

We give some examples to illustrate certain notions previously defined.

Example 11 Let $X$ be the real plane with coordinate $\left(x_{1}, x_{2}\right)$ and let $\lambda \equiv\left(\lambda_{1}, \lambda_{2}\right)$ be a given unit vector in $X$. We define a linear preference $p$ on $X$ by

$$
x \equiv\left(x_{1}, x_{2}\right) \precsim y \equiv\left(y_{1}, y_{2}\right) \text { in } p
$$

iff

$$
\langle\lambda, x\rangle \leq\langle\lambda, y\rangle,
$$


where $\langle\lambda, x\rangle \equiv \lambda_{1} x_{1}+\lambda_{2} x_{2}$ is the inner product of $\lambda$ and $x$. Then the indifference sets are the straight lines:

$$
\langle\lambda, x\rangle=\alpha ; \alpha \in A, \text { where } A \equiv(-\infty, \infty), \text { the index set. }
$$

Given a point $x=\left(x_{1}, x_{2}\right)$ in $X$, we have

$$
\begin{aligned}
I_{x} & =\{y \in X ; \quad\langle\lambda, y\rangle=\langle\lambda, x\rangle\} \\
R_{x} & =\{y \in X ; \quad\langle\lambda, y\rangle \geq\langle\lambda, x\rangle\} \\
Q_{x} & =\{y \in X ; \quad\langle\lambda, y\rangle \leq\langle\lambda, x\rangle\}
\end{aligned}
$$

where $R_{x}$ and $Q_{x}$ are closed ( $I_{x}=R_{x} \cap Q_{x}$ is consequently closed). Therefore $p$ is continuous, i.e., it respects the topology of $X$ and hence $p \in P(X)$. On the other hand, for each $\alpha \in \mathbf{R} \equiv \mathbf{A}$, let

$$
A_{\alpha}=[\alpha, \infty) \subset \mathbf{R},
$$

$\alpha=\langle\lambda, x\rangle$ and

$$
\begin{aligned}
I_{\alpha}=\{y \in X ; & \langle\lambda, y\rangle=\alpha\} \\
R_{\alpha}=\{y \in X ; & \langle\lambda, y\rangle \geq \alpha\} \\
Q_{\alpha} & =\{y \in X ; \quad\langle\lambda, y\rangle \leq \alpha\}
\end{aligned}
$$

then $I_{x}=I_{\alpha}, R_{x}=R_{\alpha}$ and $Q_{x}=Q_{\alpha}$. Clearly, $\sigma \equiv\left(X, I_{\alpha} ; A, A_{\alpha}\right)$ is the stratification corresponding to $p$.

Example 12 Let $X=[a, b] \times[-1,1]$, both $a$ and $b$ are $<-1$. We define a binary relation $p$ by $x \equiv\left(x_{1}, x_{2}\right) \precsim y \equiv\left(y_{1}, y_{2}\right)$ in $p$, iff (i) $x_{1} \leq y_{1}$, when $x_{2}=y_{2}=0$, and (ii) $\left|x_{2}\right| \leq\left|y_{2}\right|$, otherwise. Then $X$ is stratified into the points on $x_{1}$-axis and the horizontal line segments in $X$ (except the segment in $x_{1}$-axis). The index set $A=[a, b] \cup(-1,1]$. For $\alpha \in[a, b], A_{\alpha}=[\alpha, b] \cup(-1,1]$. For $\alpha \in(-1,1]$, $A_{\alpha}=(\alpha, 1]$. This is not a stratification respecting the topology of $X$ as defined in Definition 2.3.1, because (S2) is not satisfied. In fact, $R_{\alpha}$ is not closed in $X$ for $\alpha \in(a, b]$, although $\forall \alpha \in A, Q_{\alpha}$ is. Furthermore, we see that $p$ is a preference order, but $p$ is not continuous, i.e., $p \in P_{o}-P(X)$, This example shows that $P_{o} \supsetneqq P(X)$

Example 13 Let $X$ be the torus $S^{1} \times S^{1}$ where $S^{1}$ denotes a unit circle. Given the product coordinate $(\theta, \varphi)$ for each point $x$ of $X$ such that

$$
X=\{(\theta, \varphi) ; 0 \leq \theta<2 \pi, 0 \leq \varphi<2 \pi\}
$$

Define a preference order $p$ on $X$ by

$$
x \equiv\left(\theta_{o}, \varphi_{o}\right) \precsim y \equiv\left(\theta_{1}, \varphi_{1}\right) \text { iff } \theta_{o} \leq \theta_{1}
$$


Clearly $p \in P_{o}$. However, $p$ is not continuous, since at each $x=\left(\theta_{o}, \varphi_{o}\right)$, we have $R_{x}=\left\{(\theta, \varphi) \in X ; \theta_{o} \leq \theta<2 \pi\right\}$ which is not closed in $X$. Hence $p \notin P(X)$. Again, the preference $p$ does not correspond to a stratification respecting the topology of $X$. Also we remark that if one consider a unit vector field $p_{c}$ which tangents to $\theta$-curves, then $p_{c} \in P_{c}$ is a Chichilnisky's preference. Again this example shows that a local preference $p_{c}$ in $P_{c}$ may not be integrated to obtain a global preference in $P(X)$.

Example 14 Let $X$ be the 2-dimensional unit sphere $S^{2}$, i.e.,

$$
X=\left\{\left(x_{1}, x_{2}, x_{3}\right) \in \mathbf{R}^{3} ; x_{1}^{2}+x_{2}^{2}+x_{3}^{2}=1\right\}
$$

Clearly, $X$ is a differentiable manifold of dimension two. ${ }^{21}$ Define a preference order $p$ on $X$ by

$$
x \equiv\left(x_{1}, x_{2}, x_{3}\right) \precsim y \equiv\left(y_{1}, y_{2}, y_{3}\right) \text { in } p \text { iff } x_{3} \leq y_{3} .
$$

It is clear that $p \in P(X)$. There corresponds the stratification consisting of the indifference sets

$$
I_{\alpha}=\left\{x \in X ; x_{3}=\alpha\right\}, \quad \alpha=[-1,1]
$$

which are all nice differentiable curves except at the north pole and at the south pole, where $\alpha=+1$ and $\alpha=-1$ respectively.

The next proposition illustrates how the topology of $X$ is reflected on the index set $A$.

Proposition 2.3.1 Let $x_{n} \rightarrow x$ in $X$, where $x_{n} \in I_{\alpha_{n}}, x \in I_{\alpha}$, and

$$
A_{\alpha_{n}} \supset A_{\alpha_{n+1}} \supset A_{\alpha}, \quad \forall n=1,2,3, \ldots
$$

Then $\bigcap_{n=1}^{\infty} A_{\alpha_{n}}=A_{\alpha}$.

Proof See the appendix AIa.

Now we show the equivalence of a continuous preference in $P(X)$ and a stratification on $X$.

Proposition 2.3.2 Let $p$ be a continuous preference on $X$. Then $p$ defines a stratification $\sigma \equiv\left(X, I_{\alpha} ; A, A_{\alpha}\right)$, where for each $x \in I_{\alpha}$, we have $I_{\alpha}=I_{x}(p), A=X / \sim$, the quotient set defined by the equivalent relation $\sim$ which is the indifference relation given by $p$, and $A_{\alpha}=\left\{\beta \in A ; I_{\beta} \succsim I_{\alpha}\right\}$. Also, $R_{\alpha}=R_{x}(p)$ and $Q_{\alpha}=Q_{x}(p)$. Conversely, given a stratification $\sigma \equiv\left(X, I_{\alpha} ; A, A_{\alpha}\right)$, there defines a continuous preference $p$ on $X$ such that

\footnotetext{
21 A differentiable manifold of dimension $n$ is a topological space, in which a point has a neighborhood homeomorphic to the Euclidean space $\mathbf{R}^{n}$, and on which differentiable functions are consistently defined.
} 


$$
x \precsim y \text { iff } A_{\alpha} \supset A_{\beta}
$$

with $x \in I_{\alpha}, y \in I_{\beta}$ and $\alpha, \beta \in A$.

Proof See Appendix AIb.

\subsection{Singularity sets of preferences}

Definition 2.4.1 Let $f$ be a continuous real valued function on $X$. We define a preference order $p$ on $X$ by

$$
x \succsim y \text { in } p \text { iff } f(x) \geq f(y)
$$

We usually call $f$ a utility function defining the preference order $p$.

It is evident that $R_{x}=\{y \in X ; f(y) \geq f(x)\}$ and $Q_{x}=\{y \in X ; f(y) \leq f(x)\}$. $R_{x}$ and $Q_{x}$ are closed in $X$ for any $x \in X$, since $f$ is continuous on $X$. Therefore, $p$ is continuous (i.e., $p$ respects the topology of $X$ ), and $p \in P(X)$. Let $\sigma=\left(X, I_{\alpha} ; A, A_{\alpha}\right)$ be the stratification defined by $p$ as in Proposition 2.3.2 with $I_{\alpha}=\{x \in X ; f(x)=\alpha\}$. The index set $A$ is the range of the function $f$.

Definition 2.4.2 Given a preference $p \in P(X)$. Define the singular set

$$
\Lambda(p) \equiv \cup\left\{I_{x}(p) ; x \in \Lambda^{o}(p)\right\}
$$

where the interior singular set $\Lambda^{o}(p)$ is defined by

$$
\left\{x \in X ; \exists \text { neighborhood } V \text { of } x \text {, with } V \subset I_{x}(p)\right\} .
$$

Definition 2.4.3 A point $x \in \Lambda(p)$ is called a singular point of $p$. Otherwise $x$ is $a$ regular point of $p$. For $x \in \Lambda^{o}(p)$, we call $x$ an interior singular point of $p$.

Remark The singularity defined here is relative to the topological structure of the given $X$. The singular points may called topological singular points, contrast to the differentiable singular points as defined by Chichilnisky.

Remark It is more proper to call $\Lambda(p)$ the pseudo singular set, because a natural definition for singular points should be limit points of interior singular points. Not every point in $\Lambda(p)$ is a such point, since $I_{x}(p)$ may be of the shape like

$$
\left(\left[0, \frac{1}{2}\right] \times[-1,1]\right) \cup\left\{(x, 0): \frac{1}{2} \leq x \leq 1\right\} \text { in } X,
$$

where $X=[-1,1] \times[-1,1]$. But by abuse of language, we rather omit the prefix "pseudo" for simplicity. 
Example 15 In Example 14, define $p_{c} \in P_{c}$ by the unit vectors tangent to the longitudes north bound on the sphere. Then $p_{c}$ is singular at the two poles in Chichilnisky's differentiable setting. But $p$ of $P(X)$ is regular in our topological setting and $\Lambda(p)$ is empty.

We give the following two weird examples of preferences, which may occur on the basis of Lebesgue measure theory. Example 16 shows a continuous preference $p \in P(X)$ whose singular set $\Lambda(p)$ may not be closed in $X$, while Example 17a illustrates a continuous preference $p \in P(X)$ which has an indifferent set containing no interior point $\left(\Lambda^{o}(p)\right.$ is therefore an empty set), but have non-zero "area" (i.e., non-zero "Lebesgue measure"). To make our theory mathematically sound and to see the extent that our main theorem works, we have to carefully deal with these peculiar examples.

Remark A preference $p$ considered in social choice theory has usually no natural way to quantify its degree of preference in order to obtain a corresponding utility function $f$. In many examples, we use $f$ to define $p$ merely for expository convenience.

Example 16 Consider the Cantor function which is a continuous increasing function $f:[0,1] \rightarrow[0,1]$ defined by $f(x)=\frac{1}{2}$ for $\frac{1}{3} \leq x \leq \frac{2}{3} ; f(x)=\frac{1}{4}$ for $\frac{1}{9} \leq x \leq \frac{2}{9}$, $f(x)=\frac{3}{4}$ for $\frac{7}{9} \leq x \leq \frac{8}{9} ; f(x)=\frac{1}{8}$ for $\frac{1}{27} \leq x \leq \frac{2}{27}, f(x)=\frac{3}{8}$ for $\frac{7}{27} \leq x \leq \frac{8}{27}$, $f(x)=\frac{5}{8}$ for $\frac{19}{27} \leq x \leq \frac{20}{27}, f(x)=\frac{7}{8}$ for $\frac{25}{27} \leq x \leq \frac{26}{27}$; . . and so on. Let $p$ be the corresponding preference on $[0,1]$ defined by $f$, and $p \in P(X)$. We have the indifference sets

$$
I_{\frac{1}{2}}(p)=\left[\frac{1}{3}, \frac{2}{3}\right], \quad I_{\frac{1}{4}}(p)=\left[\begin{array}{ll}
\frac{1}{9}, & \frac{2}{9}
\end{array}\right], \quad I_{\frac{3}{4}}(p)=\left[\frac{7}{9}, \frac{8}{9}\right], \ldots
$$

and the interior singular set

$$
\Lambda^{o}(p)=\left(\frac{1}{3}, \frac{2}{3}\right) \cup\left(\frac{1}{9}, \frac{2}{9}\right) \cup\left(\frac{7}{9}, \frac{8}{9}\right) \cup \cdots,
$$

with the singular set identical with the Cantor set, i.e.,

$$
\Lambda(p)=\left[\frac{1}{3}, \frac{2}{3}\right] \cup\left[\frac{1}{9}, \frac{2}{9}\right] \cup\left[\frac{7}{9}, \frac{8}{9}\right] \cup \cdots
$$

But the closure of $\Lambda^{o}(p)$ is

$$
\overline{\Lambda^{o}(p)}=[0,1]=X,
$$

which is different from $\Lambda(p)$, and therefore the singular set $\Lambda(p)$ is not closed in $X$. In this example, although the boundary $\partial I_{x}(p)$ of the indifference set $I_{x}(p)$ for each given $x \in \Lambda(p)$ contains exactly two points, $\partial \Lambda(p)$ has uncountable set of points.

Example $17 a$ Let $X$ be the square $I^{2}=[0,1] \times[0,1]$. We may enumerate the set of all rational points of $X$, i.e., the points in $X$ with both the two coordinates $x_{1}$ and $x_{2}$ rational into 


$$
\left\{r_{1}, r_{2}, r_{3}, \ldots, r_{n}, \ldots\right\}
$$

Given $\varepsilon$ with $0<\varepsilon<1$, define $Z_{\varepsilon} \equiv X-\bigcup_{k=1}^{\infty} B\left(r_{k}, \sqrt{\varepsilon / 2^{k+2}}\right)$ where $B\left(x_{o}, \rho\right)$ denotes the open disk $\left\{x \in \mathbf{R}^{2} ;\left|x-x_{o}\right|<\rho\right\}$.

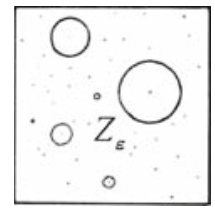

Fig. 9

Define a continuous preference order $p_{\varepsilon}$ on $X$ by

$$
y \succsim x \text { in } p_{\varepsilon} \text { iff } \operatorname{dist}\left(y, Z_{\varepsilon}\right) \leq \operatorname{dist}\left(x, Z_{\varepsilon}\right)
$$

where $\operatorname{dist}(x, A)$ denotes the Euclidean distance from a point of $x$ to a closed set $A$ in $\mathbf{R}^{2}$. Since dist $\left(x, Z_{\varepsilon}\right)$ is a continuous function of $x$, the preference $p_{\varepsilon}$ is continuous. For any $x \in Z_{\varepsilon}$, the indifference set $I_{x}\left(p_{\varepsilon}\right)$ is identical with $Z_{\varepsilon}$ and is closed in $X$. Since all indifferent sets, including $Z_{\varepsilon}$, contain no open subset of $X, \Lambda^{o}\left(p_{\varepsilon}\right)$ is empty and the preference $p_{\varepsilon}$ is topologically regular. However, it is singular with respect to the structure of Lebesgue measure. The relativist viewpoint of the notion of singularity relative to the mathematical structure under consideration will be explained in Sect. 2.6.

\subsection{Stratification topology $\mathfrak{s}$}

The equivalence of a preference and a stratification established in Proposition 2.3.2 emphasizes the importance of the stratification structure in setting a topology on the preference space. We introduce for $P(X)$ the stratification topology $\mathfrak{s}$, so that the corresponding convergence of a sequence of preferences respects the stratification structure, in the sense that the Criterion A, mentioned in (II) of Introduction, is fulfilled. To define $\mathfrak{s}$, we use the language of open sets, regarding a topology as a family of open sets satisfying: (a) any union of open sets is open, (b) a finite intersection of open sets is open, and (c) the empty set and the total set is open (see Kelly 1955).

Definition 2.5.1 Let $\mathfrak{\Im}_{O}$ be the zero order topology of $P(X)$ defined by the subbase ${ }^{22}$

$$
\mathcal{B}_{o} \equiv\{\Gamma(K, L) ; K, L \text { are compact sets } \subset X\}
$$

\footnotetext{
22 Any open set of $P(X)$ defined by the topology $\Im_{O}$ is a union of members of $\widehat{B}_{O}$ where $\widehat{B}_{O} \equiv\{$ finite intersections of members of $\left.B_{O}\right\}$, or equivalently, any neighborhood of a preference $p$ in $P(X)$ defined by the topology contains a set of the form $\cap\left\{\Gamma\left(K_{i}, L_{i}\right) ; i=1,2, \ldots, m\right\}$, where $\Gamma\left(K_{i}, L_{i}\right) \in B_{o}$ and $m$ is a natural number. See also Kelly (1955).
} 
where $\Gamma(K, L) \equiv\{p \in P(X) ; K \prec L$ in $p\}$. Let

$$
\mathcal{B}_{1} \equiv\{\Omega(J, W) ; J \subset W, J \text { is non-empty and compact, } W \text { is open in } X\}
$$

where $\Omega(J, W) \equiv\left\{p \in P(X) ; J \subset \Lambda^{o}(p) \subset \Lambda(p) \subset W,\right\}$ and $\Lambda(p)$ is the singular set with $\Lambda^{o}(p)$ the interior singular set (see Definition 2.4.2).

Definition 2.5.2 The topology $\mathfrak{s}$ defined by the subbase $\mathcal{B}_{o} \cup \mathcal{B}_{1}$, is called the stratification topology of $P(X)$ or called the first order topology of $P(X)$. Given a sequence $p_{n}$ in $P(X)$ and $p_{o} \in P(X)$, we say $p_{n}$ converges to $p_{o}$ in $\mathfrak{\Im}$, if $\forall T \in \Im$ with $p_{o} \in T$, there exists an integer $N$ such that $p_{n} \in T, \forall n>N$. The convergence is usually denoted by

$$
p_{n} \rightarrow p_{o} \text { in } \mathfrak{\Im} .
$$

Since $\mathcal{B}_{o} \cup \mathcal{B}_{1}$ generate $\mathfrak{s}$, a criterion for $p_{n} \rightarrow p_{o}$ in $\Im$ is that: $\forall \Gamma(K, L)$ and $\forall \Omega(J, W)$ with $K, L, J$ compact, $J \neq \phi$, and $W$ open in $X$, if $p_{o} \in \Gamma(K, L) \cap$ $\Omega(J, W)$, then there exists an integer $N$ such that $p_{n} \in \Gamma(K, L) \cap \Omega(J, W), \forall n>N$.

Remark Note that $\mathfrak{\Im}_{o}$ corresponds to the condition (A1) of Criterion A in Introduction of this paper. And the stratification topology $\mathfrak{I}$ is designed to fit the notion of the convergence of preferences satisfying Criterion A.

Proposition 2.5.1 Let $p_{n} \rightarrow p_{o}$ in $\mathfrak{s}$. Then the statement $(\boldsymbol{H})$ defined by

$$
\begin{aligned}
x \succ y \text { in } p_{o} \Rightarrow & \exists \text { neighborhoods } U, V \text { of } x, y \text { respectively } \\
& \text { and } \exists N \text { such that } U \succ V \text { in } p_{n}, \forall n>N .
\end{aligned}
$$

is valid.

Proof By Proposition 2.2.2, $\exists$ neighborhoods $U_{1}, V_{1}$ of $x, y$ respectively such that

$$
U_{1} \succ V_{1} \text { in } p_{o}
$$

Since $X$ is locally compact, there exists $\bar{U}$ and $\bar{V}$, compact neighborhoods of $x$ and $y$ respectively, such that $\bar{U} \subset U_{1}$ and $\bar{V} \subset V_{1}$ with $U, V$ open neighborhoods of $x y$ in $X$. Clearly $p_{o} \in \Gamma(\bar{V}, \bar{U})$. By the definition of $\Im, \exists N$ such that $p_{n} \in \Gamma(\bar{V}, \bar{U}), \forall n>N$. In particular,

$$
U \succ V \text { in } p_{n}, \forall n>N,
$$

as required.

If the premise of Proposition 2.5.1 is replaced by $p_{n} \rightarrow p_{o}$ in $\mathfrak{\Im}_{o}$, the proposition is still valid, because the above proof involves only open sets generated by $\Gamma(*, *)$ in $\mathfrak{\Im}_{o}$. Furthermore, if the statement $(\mathbf{H})$ is satisfied, then $p_{n} \rightarrow p_{o}$ in $\mathfrak{\Im}_{o}$.

The last Proposition 2.5.1 says that the topology $\mathfrak{s}$ as well as $\mathfrak{\Im}_{o}$ fits the condition (A1) of Criterion A. In fact, the indifference sets $I_{\alpha}$ and $I_{\beta}$ are compact, since they 
are closed in compact $X$. By using $(\mathrm{H})$ and the standard finite covering argument on compact sets, (A1) is proved as claimed.

From the definition of $\mathcal{B}_{1}$, it is straightforward that

$$
\left(\mathrm{A} 2^{\prime}\right) \Lambda\left(p_{n}\right) \text { tends to } \Lambda\left(p_{o}\right) \text {. }
$$

Furthermore, $\mathfrak{\Im}$ also fits the condition (A2) of Criterion A. The proof will be seen later in Theorem 1 of Sect. 3.

The following proposition is almost a logical equivalence of the last proposition.

Proposition 2.5.2 Let $p_{n}, p_{o} \in P(X)$. Then $p_{n} \rightarrow p_{o}$ in $\mathfrak{\Im}_{o}$, if and only if for each pair $x$ and $y$ in $X$,

$$
\text { (S) } \Rightarrow x \succsim y \text { in } p_{o} \text {, }
$$

where the condition $(\boldsymbol{S})$ is defined by

$$
\exists x_{n} \rightarrow x \text { and } y_{n} \rightarrow y \text { in } X \text { with } x_{n} \succsim y_{n} \text { in } p_{n}, \forall n>\text { some } N
$$

Note that the assumption that $p_{n}$ and $p_{o}$ belong to $P(X)$ is necessary. If one tries to use the condition $(\boldsymbol{S})$ to construct a binary relation $p_{o}$ with $p_{n}$ given, the resultant $p_{o}$ may not be transitive. This remark is significant which will be seen later on. We show this observation by the following example.

Example 18 Let $p_{n}$ be the preferences defined by the utility functions $f_{n}$ on $\mathbf{R}^{1}$ which are given by

$$
f_{n}(x)= \begin{cases}-x, & x \leq 0 \\ x / n & x>0\end{cases}
$$

and illustrated in Fig. 10 below. Using (S), one can construct a binary relation $p_{o}$. Given $x>y>0$ in $\mathbf{R}^{1}$. By (S), one can check that $x \succsim y$ inp $p_{o}$, but not $y \succsim x$ in $p_{o}$. Hence $x \succ y$ in $p_{o}$. On the other hand, we may choose $z_{n} \in \mathbf{R}^{1}$ with $z_{n}<0$ and $z_{n} \rightarrow 0$, such that $f_{n}\left(z_{n}\right) \geq f_{n}(x)$, i.e., $z_{n} \succsim x$ in $p_{n}$. By (S), we have $0 \succsim x$ in $p_{o}$. Similarly, we have $y \succsim 0$ in $p_{o}$, while choosing $z_{n}^{\prime}<0$ such that $f_{n}\left(z_{n}^{\prime}\right) \leq f_{n}(y)$. But we have known that $x \succ y$ in $p_{o}$. Hence the transitivity is false for $p_{o}$.And therefore the hypothesis that $p_{o} \in P(X)$ in Proposition 2.5.2 is essential.

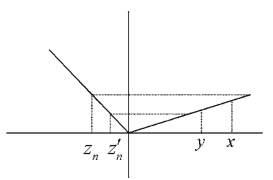

Fig. 10 
Remark For a preference $p \in P(X)$, consider the graph

$$
G(p) \equiv\{(x, y) \in X \times X ; x \succsim y \text { in } p\}
$$

Let $p_{n} \in P(X)$ with $\lim G\left(p_{n}\right)=$ a subset $G_{o}$. Example 18 shows that $G_{o}$ may not correspond to a preference $p_{o}$ in $P(X)$. However, if $p_{n}$ and $p_{o} \in P(X)$, then by Proposition 2.5.2 it is easy to see that $p_{n} \rightarrow p_{o}$ in $\mathfrak{\Im}_{o}$ iff $\lim G\left(p_{n}\right)=G\left(p_{o}\right)$.

Example 19 Consider the linear preference $p_{n}$ on $\mathbf{R}^{2}$ defined in Example 11 by letting $\lambda_{n}=\left(\cos \frac{\pi}{n}, \sin \frac{\pi}{n}\right)$ and consider $p_{o}$ defined by $\lambda_{o}=(1,0)$. Then $p_{n} \rightarrow p_{o}$ in $\mathfrak{s}$ as well as in $\mathfrak{\Im}_{o}$, when $n \rightarrow \infty$.

It is evident that in Examples 1, 2, 3, 4 and 7, the preferences $p_{n}, p_{o} \in P(X)$, and $p_{n} \rightarrow p_{o}$ in $\mathfrak{\Im}$ and therefore in $\mathfrak{\Im}_{o}$. However, in Example 5 and $8, p_{\varepsilon}$ tends to $p_{o}$ in $\mathfrak{s}_{O}$ but not in $\mathfrak{\Im}$..

For $p_{n}$ convergent to $p_{o}$, the inferior sets or the preferred sets of $p_{n}$ may not converge to those of $p_{o}$, although the singular sets do.

Example 20 Let $X=[-1,3]$ and let the preference $p_{n}$ be defined by the utility function $f_{n}$, which is constructed as follows (see Fig. 11). Put $f_{n}\left(\frac{1}{n}\right)=f_{n}(1)=$ $f_{n}(3)=0, f_{n}(-1)=1$ and $f_{n}(2)=-1$, such that $f_{n}$ is linear on each of the intervals $\left[-1, \frac{1}{n}\right],\left[\frac{1}{n}, 1\right],[1,2]$ and $[2,3]$. Let $f_{o}(x)=\lim _{n \rightarrow \infty} f_{n}(x)$ and $p_{o}$ be defined by $f_{o}$. Then $\Lambda^{o}\left(p_{o}\right)=(0,1)$ and $\Lambda^{o}\left(p_{n}\right)=\left(\frac{1}{n}, 1\right)$. Also, $\Lambda\left(p_{o}\right)=[0,1]$ and $\Lambda\left(p_{n}\right)=\left[\frac{1}{n}, 1\right]$. Given $K \subset(0,1) \subset[0,1] \subset W$, where $K$ is a compact set and $W$ is open in $X$, we have $p_{o} \in \Omega(K, W) \in \mathcal{B}_{1}$. For $n$ sufficiently large, we see that

$$
K \subset\left(\frac{1}{n}, 1\right) \subset\left[\frac{1}{n}, 1\right] \subset W
$$

which yields that $p_{n} \in \Omega(K, W)$, for large $n$. It is also clear that given $\Gamma(J, L) \in \mathcal{B}_{o}$, $p_{o} \in \Gamma(J, L)$ implies $p_{n} \in \Gamma(J, L)$ for large $n$. Therefore,

$$
p_{n} \rightarrow p_{o} \text { in } \Im
$$

However, at $x=0$ the inferior set $Q_{x}^{\prime}\left(p_{n}\right)=(0,3]$, while $Q_{x}^{\prime}\left(p_{o}\right)=(1,3)$. Therefore, $Q_{x}^{\prime}\left(p_{n}\right)$ does not tend to $Q_{x}^{\prime}\left(p_{o}\right)$. However, at $x=0, Q^{\prime}\left(p_{n}\right)-\Lambda\left(p_{n}\right)=\left(0, \frac{1}{n}\right) \cup(1,3]$ tends to $Q_{x}^{\prime}\left(p_{o}\right)-\Lambda\left(p_{o}\right)=(1,3)$.

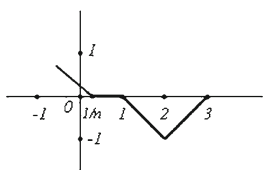

Fig. 11

In general, for $p_{n} \rightarrow p_{o}$ in $\Im$, the inferior set $Q_{x}^{\prime}\left(p_{n}\right)$ may not tend to $Q_{x}^{\prime}\left(p_{o}\right)$. But the set $Q_{x}^{\prime}\left(p_{n}\right)-\Lambda\left(p_{n}\right)$ does tend to $Q_{x}^{\prime}\left(p_{o}\right)-\Lambda^{o}\left(p_{o}\right)$ in most reasonable cases. This is an important observation in proving the lifting theorem (Theorem 2) which basically supports the main theorem (Theorem 3). 


\subsection{Singularity relative to given structures}

In this subsection, we introduce the concept of singularity/regularity of preferences in a general way. Let $X$ be a general space with various structures.

The concept of singularity/regularity of preferences is related to the structure of $X$ under consideration. We will define them in set theoretic structure, in topological structure, in manifold structure or in differentiable structure, as well as in measure structure. Although the notion of regularity has a different description, each formed by the concerned structure, it has a core characteristic that when the preference order gradually increases, the corresponding stratification (or more precisely, the family of the indifference sets) covers the alternative space $X$ as evenly and smoothly as the relative structure could detect and describe. Singularity occurs otherwise.

Definition 2.6.1 Let $X$ be a pure set, a preference order $p$ on $X$ is singular in the pure set structure if there exists two distinct $x$ and $y$ such that $x \sim y$ (Call such $x$ a singular point of $p$ ). Otherwise, $p$ is regular. The union of the indifference sets of the singular points is the singular set.

Definition 2.6.2 Let $X$ be a connected topological space or (even a continuum). A preference $p$ on $X$ is singular in the topological structure if there exists a point $x$ in $X$ and a neighborhood $V_{x}$ of $x$ such that $V_{x} \subset I_{x}(p)$. Otherwise, $p$ is regular in the topological structure.

Example 21 (a) Let $p$ be given by the utility function $f$ with $f(x)=A x_{1}^{2}+$ $2 B x_{1} x_{2}+C x_{2}^{2}$ for $x=\left(x_{1}, x_{2}\right) \in X \equiv R^{2}$ with $B^{2}-A C \neq 0$, then $p$ is regular in the (canonical) topological structure, although we will see later that it is singular both in the manifold structure and in the differentiable structure.

(b) If $p$ is defined by the utility function:

$$
f\left(x_{1}, x_{2}\right)= \begin{cases}x_{1}, & \text { if } x_{1} \leq 0 \\ 0, & \text { if } 0<x_{1}<1 \\ x_{1}-1, & \text { if } x_{1} \geq 1\end{cases}
$$

then $p$ is singular in the topological structure.

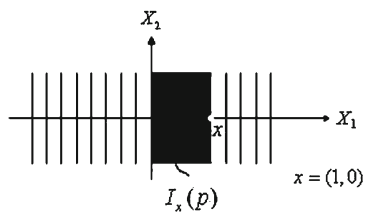

Fig. 12

Definition 2.6.3 Let $X$ be a connected topological manifold of dimension $n$. A preference is regular in the manifold structure if each indifferent set $I_{x}$ of a point $x$ in $X$ is a topological $n-1$ dimensional submanifold of $X$ and any neighborhood of $x$ contains a smaller neighborhood $V_{x}$ such that $V_{x}-I_{x}$ has exactly two components, one in the preferred set $R_{x}^{\prime}$ and the other in the inferior set $Q_{x}^{\prime}$ unless $x$ is a boundary 
point of $X$ when $X$ has boundary. If $p$ is not regular, call $p$ singular in the manifold structure.

Example 22 Let $p$ be given by the utility function

$$
f\left(x_{1}, x_{2}\right)=x_{1}^{n}, \quad x=\left(x_{1}, x_{2}\right) \in R^{2} \equiv X
$$

Then $p$ is regular in the (canonical) manifold structure for $n$ odd, and singular for $n$ even. However, it is regular in the topological structure for any $n$.

Definition 2.6.4 Let $X$ be a connected differentiable manifold of dimension $\dot{n}$. A preference $p$ on $X$ is regular in the differentiable structure if $p$ is regular in the manifold structure and each $I_{x}$ is a differentiable submanifold of dimension $n-1$. Otherwise $p$ is singular in the differentiable structure.

Example 23 Let $p$ on $X=\mathbf{R}^{2}$ be defined as follows (see Fig. 13)

(i) $\forall x=(a, 0)$ with $a \in \mathbf{R}, I_{x}=\{(a(1+|t|), t) ; t \in \mathbf{R}\}$

(ii) For $x=(a, 0)$ and $y=(b, 0), x>y$ in $p$ iff $a>b$.

Then $p$ is regular in the (canonical) manifold structure of $\mathbf{R}^{2}$, but singular in the (canonical) differentiable structure of $\mathbf{R}^{2},{ }^{23}$ since $I_{x}$ is non-differentiable on $x_{2}=0$.

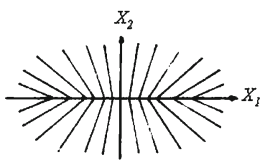

Fig. 13

It follows immediately that a preference singular in a topological structure is singular in the corresponding manifold structure. And if $p$ is singular in a manifold structure, then it is singular in the related differentiable structures. Finally, we consider the singularity in a measure structure.

Definition 2.6.5 Let $X$ be a locally compact Hausdorff space with a measure structure $\mu$. A preference $p$ on $X$ is regular in the measure structure if each $I_{x}$ has zero measure, i.e., $\mu\left(I_{x}\right)=0$. Otherwise $p$ is singular in the measure structure.

If $X$ has a measure structure $\mu$, to let a preference under consideration make sense we should assume that the preference respects the measure structure $\mu$, in the way that the graph $G \subset X \times X$ which defines the preference is $\mu$-measurable in $X \times X$. This is equivalent to the assumption that each indifference set of the preference is $\mu$-measurable, in which case we call that the preference is $\mu$-measurable.

Definition 2.6.6 Let $X=\mathbf{I}^{n}$. A set $E$ in $\mathbf{I}^{n}$ is called an $n$-rectangle, if $E=\left[a_{1}, b_{1}\right] \times$ $\left[a_{2}, b_{2}\right] \times \cdots \times\left[a_{n}, b_{n}\right]$. Let the area of $E$ is defined by

$$
\mu(E):=\left(b_{1}-a_{1}\right)\left(b_{2}-a_{2}\right) \cdots\left(b_{n}-a_{n}\right) .
$$

\footnotetext{
23 The differentiable structure is the canonical one of $\mathbf{R}^{2}$.
} 
For a set $D$ in $\mathbf{I}^{n}$, the outer Jordan measure $\mu^{*}(D)$ is defined by

$$
\mu^{*}(D):=\inf \left\{\Sigma_{i=1}^{k} \mu\left(E_{i}\right) ; E_{1} \cup \cdots \cup E_{k} \supset D, k \in \mathbf{N}, E_{i}=n \text {-rectangle, } \forall i\right\}
$$

where $\mathbf{N}$ is the set of the natural numbers. Similarly, the inner Jordan measure $\mu_{*}(D)$ is defined by

$$
\mu_{*}(D) \equiv \sup \left\{\Sigma_{i=1}^{k} \mu\left(E_{i}\right) ; E_{1} \cup \cdots \cup E_{k} \subset D, k \in \mathbf{N}, E_{i}=n \text {-rectangle, } \forall i\right\}
$$

If $\mu^{*}(D)=\mu_{*}(D)$, we say $D$ is Jordan measurable and define

$$
\mu(D) \equiv \mu^{*}(D)=\mu_{*}(D)
$$

which is called the Jordan measure of $D$, or the $n$-area of $D$ as the common name.

It is easy to see the following

Proposition 2.6.1 If $D$ in $\mathbf{I}^{n}$ is Jordan measurable, then

$$
\mu(\partial(D))=0 .
$$

In the following section (Sect. 3), we consider the alternative space $X$ with the Jordan measure structure $\mu$ and correspondingly make a further assumption that any of the preferences under consideration is Jordan measurable.

Definition 2.6.7 Let $X \equiv \mathbf{I}^{n}$ and let the Jordan measure structure on $\mathbf{I}^{n}$ be denoted by $\mu$. A preference in $P(X)$ is called normal, if it is also $\mu$-measurable. In other words, a normal preference is a continuous and $\mu$-measurable preference order. The totality of normal preferences is denoted by $P(X, \mu)$, meaning it respect the topological structure of $X$ and the Jordan measure $\mu$. The space $P(X, \mu)$ is the preference space that we consider in our main theorem, and it will be abbreviated by $P$ in notation.

Example $17 b$ In Example 17aa, we have noted that $Z_{\varepsilon}=I_{x}\left(p_{\varepsilon}\right)$ for $x \in Z_{\varepsilon}$. It is clear that $Z_{\varepsilon}$ is not Jordan measurable. Hence $p_{\varepsilon}$ is not $\mu$-measurable and hence not normal. Thus $p_{\varepsilon} \in P(X)-P$. Let $m$ be the "Lebesgue measure" on $\mathbf{R}^{2}$ which is a more sophisticated notion of the "area" than Jordan measure. It can be shown that $Z_{\varepsilon}$ is Lebesgue measurable and hence $p_{\varepsilon}$ is $m$-measurable. Evidently, $Z_{\varepsilon}$ has the Lebesgue measure

$$
m\left(Z_{\varepsilon}\right) \geq 1-\frac{\pi}{4} \varepsilon>1-\varepsilon>0,
$$

although it has no interior point. Let $x \in Z_{\varepsilon}$, we see that $I_{x}\left(p_{\varepsilon}\right)=Z_{\varepsilon}$ and relative to the Lebesgue measure structure, $p_{\varepsilon}$ is singular. The singular set of $p_{\varepsilon}$ is $I_{x}\left(p_{\varepsilon}\right)=$ $Z_{\varepsilon} \neq \phi$. Since the interior Int $I_{x}\left(p_{\varepsilon}\right)$ is empty, we have $\partial I_{x}\left(p_{\varepsilon}\right)=I_{x}\left(p_{\varepsilon}\right)$, and

$$
m\left(\partial I_{x}\left(p_{\varepsilon}\right)\right)=m\left(I_{x}\left(p_{\varepsilon}\right)\right)=m\left(Z_{\varepsilon}\right)>0 .
$$

Note that Proposition 2.6.1 is not valid for Lebesgue measure. 
All the continuous preferences (of $P(X)$ ), previously given as examples in this paper, are $\mu$-measurable and therefore normal, except the one in Example 17a.

Certainly, we may develop the theory using the Lebesgue measure to replace the Jordan measure, so that the weird example of the preference described in Example 17a is included.

However, it seems not necessary at the moment to do that way, because on the one hand, it increases a great amount of technical complication, and on the other hand, the classical notion of area, i.e., the Jordan measure, is sufficient to provide us a tool to see the essence of social choice theory. ${ }^{24}$

Note that the key difference between the classical Jordan measure theory and the modern Lebesgue measure theory is that the number of the n-rectangles $E_{i}$ 's used to define the outer (inner) measures is finite for Jordan, while it is infinite for Lesbegue.

\section{The theorems}

\subsection{Uniform approximation theorem}

We have claimed in Sect. 2.4 that our stratification topology $\mathfrak{s}$ on $X$ does respect the stratification structure in the sense that the two conditions (A1) and (A2) of Criterion A are satisfied. In Proposition 2.5.2, the condition (A1) has been proved. We now show the condition (A2).

Theorem 1 (Uniform approximation theorem) Let $p_{n} \rightarrow p_{o}$ in $\mathfrak{s}$. Given $x \in X$, if

$$
I_{x}\left(p_{o}\right) \subset W
$$

for some open set $W$, then $\exists N$ and $\exists$ a compact neighborhood $V$ of $x$ such that $I_{v}\left(p_{n}\right) \subset W, \forall n>N$ and $\forall v \in V$. Furthermore, if

$$
x \in K \subset \text { Int } I_{x}\left(p_{o}\right)
$$

for some compact connected set $K$, then $\exists N_{1}$ and $\exists$ a compact neighborhood $V_{1}$ of $x$ such that $K \subset$ Int $I_{v}\left(p_{n}\right), \forall n>N_{1}$ and $\forall v \in V_{1}$.

Proof See Appendix (AII).

Corollary 3.1.1 Given $p_{n}, p_{o} \in P(X)$, we have $p_{n} \rightarrow p_{o}$ in $\Im$ if and only if $p_{n}$ and $p_{o}$ satisfy Criterion $\boldsymbol{A}$.

Proof See Appendix (AIIa).

\footnotetext{
24 This is similar to the historical development in the centuries from Fermat to Cantor. During that period, the classical integration theory, in which the number of discontinuous points of a function is assumed finite, sufficed to handle the problems that men ever encountered in that time. After a series of paradoxes occurred on the subjects of Fourier series and the function theory, the rigorous real number system was invented in the middle nineteenth century, and the studies of Lebesgue integration theory become inevitable. Since then more complicate functions (e.g., Cantor's function given in Example 16) were found and have to be tackled.
} 
The condition that $K$ be connected in Theorem 1 is essential, as seen in the following example. It means that the approximation of indifferent sets in Criterion (A2) is "componentwise".

Example 24 (see Fig. 14) Let $X=[-2,2] \times[0,1] \subset \mathbf{R}^{2}$ and let $p_{n}$ and $p_{o}$ be defined by the utility functions $f_{n}$ and $f_{o}$ respectively, where $f_{n}$ and $f_{o}$ are given by

$$
f_{n}(-2)=0=f_{n}(-1), \quad f_{n}(0)=1, \quad f_{n}(1)=\frac{1}{n}=f_{n}(2)
$$

and

$$
f_{o}(-2)=0=f_{o}(-1), \quad f_{o}(0)=1, \quad f_{o}(1)=0=f_{o}(2),
$$

such that $f_{n}(x)$ and $f_{o}(x)$ are linear on the four intervals $[-2,-1],[-1,0],[0,1]$ and $[1,2]$.

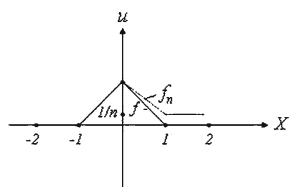

Fig. 14

Let $x=-\frac{3}{2}$. Clearly, $I_{x}\left(p_{o}\right)=[-2,-1] \cup[1,2]$, while $I_{x}\left(p_{n}\right)=[-2,-1]$. We see that at the singular point $x, I_{x}\left(p_{n}\right)$ does not tend to $I_{x}\left(p_{o}\right)$, although $p_{n} \rightarrow p_{o}$ in $\mathfrak{\Im}$. In this example, if we let $K=\left[-\frac{5}{3},-\frac{4}{3}\right] \cup\left[\frac{4}{3}, \frac{5}{3}\right]$ which is not connected, then the second statement of Theorem 1 would be false. However, the singular set $\Lambda\left(p_{n}\right)$ of $p_{n}$ still converges to the singular set $\Lambda\left(p_{o}\right)$ of $p_{o}$.

Also the following Example 25 shows that the local connectedness ${ }^{25}$ of $X$ is essential in Theorem 1.

Example 25 (See Fig. 15) Let

$$
X=\left\{\left(t, \sin \frac{1}{t}\right) \in \mathbf{R}^{2} ; 0 \leq t \leq 1\right\} \cup\{(0, s) ;-1 \leq s \leq 1\} .
$$
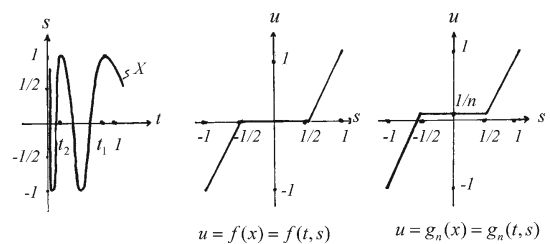

Fig. 15

\footnotetext{
25 By $X$ locally connected, we mean $\forall x \in X, \forall$ neighborhood $U$ of $x$ the component of $U$ containing $x$ is also a neighborhood of $x$.
} 
Notice that $X$ is connected but not locally connected. We define continuous functions $f$ and $f_{n}$ on $X$ as follows. Given $x=(t, s) \in X$,

$$
f(x)=\left\{\begin{array}{cc}
0 ; & s \in\left[-\frac{1}{2}, \frac{1}{2}\right] \\
2 s-1 ; & s \in\left[\frac{1}{2}, 1\right] \\
2 s+1 ; & s \in\left[-1,-\frac{1}{2}\right]
\end{array}\right.
$$

and

$$
f_{n}(x)=\left\{\begin{array}{cc}
f(x) ; & t \leq t_{n} \\
g_{n}(x) ; & t>t_{n}
\end{array}\right.
$$

where $t_{n}$ 's are the maximal points of $h(t)=\sin \frac{1}{t}$, ordered as $1>t_{1}>t_{2}>\cdots>$ $t_{n}>\cdots \rightarrow 0$. and

$$
g_{n}(x)= \begin{cases}1 / n ; & s \in[-1 / 2,1 / 2] \\ (2 s-1)(1-1 / n)+1 / n ; & s \in[1 / 2,1] \\ (2 s+1)(1+1 / n)+1 / n ; & s \in[-1,-1 / 2] .\end{cases}
$$

Let the preferences $p_{n}$ and $p$ be defined on $X$ correspondingly by the utility functions $f_{n}$ and $f$. Denote the set $\{(t, s) \in X ;-1 / 2 \leq s \leq 1 / 2\}$ by $\Sigma$. Then the singular sets are

$$
\Lambda\left(p_{n}\right)=\Lambda\left(p_{o}\right)=\Sigma
$$

Evidently, $p_{n} \rightarrow p_{o}$ in $\Im$. Choose $x=(0,0)$. We have

$$
I_{x}\left(p_{o}\right)=\Sigma, \text { and } I_{x}\left(p_{n}\right)=\left(\Sigma \cap A_{n}\right) \cup\{\text { isolated points }\}
$$

where $A_{n}=\left\{(t, s) \in X ; t \leq t_{n}\right\}$. Evidently, $I_{x}\left(p_{n}\right)$ does not tend to $I_{x}\left(p_{o}\right)$ as $n \rightarrow \infty$. Let $K$ be the connected set $\{(0, s) \in X ; s \in[-1 / 3,1 / 3]\}$. Then $x \in K \subset \operatorname{Int} I_{x}\left(p_{o}\right)$. Given any neighborhood $V$ of $x$ in $X$, there must exist $v=(\tau, \sigma) \in V \cap \Sigma$ with $\tau>0$. Choose $n_{o}$ such that $t_{n_{o}}<\tau$. Then $\forall n>n_{o}, I_{v}\left(p_{n}\right)=\left(\Sigma \cap A_{n}^{c}\right) \cup$ isolated points\} where $A_{n}^{c}=\left\{(t, s) \in X ; t>t_{n}\right\}$. But $K \cap A_{n}^{c}=\phi$. Therefore $K \cap I_{v}\left(p_{n}\right)=\phi$. Then second statement of Theorem 1 is false in this example, where $X$ is not locally connected.

\subsection{The main theorem}

We first define the space of utility functions and its topology.

Definition 3.2.1 Let $C^{o}(X)$ denote the space of all real-valued continuous functions defined on $X$, equipped with the compact-open topology. More precisely, the topology of $C^{o}(X)$ is generated by the family

$$
\{\mathcal{F}(K, U) ; K \text { : compact } \subset X, U \text { : open in } \mathbf{R}\}
$$


where $\mathcal{F}(K, U) \equiv\left\{f \in C^{o}(X) ; f(K) \subset U\right\}$. If $X$ is considered as a continuum alternative space, we say $C^{o}(X)$ the space of utility functions on $X$.

It is easy to see that the compact open topology of $C^{o}(X)$ corresponds to the uniform convergence on compact sets for functions in $C^{o}(X)$. Therefore it is the most natural and common topology.

In general, a function $f \in C^{o}(X)$ defines a continuous preference $p$ on $X$, such that $f$ is a utility function in $x$ corresponding to $p$. The following lifting theorem shows the reverse way that there exists a map $\xi$ which assigns to each preference $p$ in $P$ a utility function $f$ in $C^{o}(X)$ "continuously", relative to the topology $\Im$ of $P$ and the compactopen topology of $C^{o}(X)$. The lifting theorem is crucial to establish our main theorem.

Theorem 2 (Lifting theorem) Let

$$
\xi: P \rightarrow C^{o}(X)
$$

be defined by $\xi(p)=f, \forall p \in P$, with

$$
f(x) \equiv \mu\left(Q_{x}^{\prime}(p)-\Lambda(p)\right)+1>0 .
$$

Then $f$ is continuous and hence $\in C^{o}(X)$. Furthermore, $\xi$ is continuous.

(Recall that the preference space $P$ is the totality of all continuous $\mu$-measurable preference orders on $X$, where $\mu$ denotes the Jordan measure meaning "the $n$-dimensional area” . Also recall that $Q_{x}^{\prime}(p)$ is the inferior set of $p$ at $x \in X$, and $\Lambda(p)$ the singular set of $p$ in $X$.)

Lemma A Let $p \in P(X)$, then

$$
\text { (i) } \Lambda^{o}(p)=\operatorname{Int} \Lambda(p)
$$

and therefore

(ii) $\partial \Lambda(p) \supset \cup_{i=1}^{\infty} \partial\left(I_{x_{i}}(p)\right)$.(Recall that $\left.\Lambda(p)=\cup_{i=1}^{\infty} I_{x_{i}}(p)\right)$.

Furthermore, when $\Lambda(p)$ is closed in $X$, we have

$$
\text { (iii) } \partial \Lambda(p)=\cup_{i=1}^{\infty} \partial\left(I_{x_{i}}(p)\right) \text {. }
$$

For the proof of Lemma A, see Appendix IIIa. Note that (iii) is not true for the Cantor preference given in Example 16. The existence of such examples show that the statements are not formally trivial.

Based on Lemma A, we can prove now the lifting theorem. It is remarked in Example 20 that the inferior set $Q_{x}^{\prime}(p)$ may not be continuous in $p$, although it is uppersemi continuous. The idea of the proof of lifting theorem is using the lemma to show that

$$
\mu\left(Q_{x}^{\prime}(p)-\Lambda(p)\right)
$$

is continuous in $p$, which then implies the continuity of the map $\xi$. 
Proof of Theorem 2 (See Appendix IIIb).

Definition 3.2.2 By a social utility map relative to a given continuum alternative space $X$ of $N$ individuals, we mean a continuous map

$$
U: P^{N} \rightarrow C^{o}(X)
$$

where $P^{N}$ denotes the product space of $N$ copies of preference space $P$ on $X$, equipped with the product topology of $\mathfrak{I}$.

It is now straightforward to show the main theorem.

\section{Theorem 3 (Existence theorem)}

There exist infinitely many continuous social utility maps which are anonymous, and satisfy the strong Pareto condition (and hence respect unanimity).

Proof Let $U$ be the composition map $G_{v, \alpha} \circ \xi^{N}$, i.e.,

$$
U: P^{N} \stackrel{\xi^{N}}{\longrightarrow}\left(C_{+}^{o}(X)\right)^{N} \stackrel{G_{v, \alpha}}{\longrightarrow} C_{+}^{o}(X) \subset C^{o}(X)
$$

where the map $\xi$ is given by the lifting theorem, and $\xi^{N}$ is the product map of $N$ copies of $\xi$. The space $C_{+}^{o}(X)$ denotes the subspace of $C^{o}(X)$ which consists of only the positive-valued functions in $C^{o}(X)$, and has the subspace topology. Given $v$ a positive integer and $\alpha$ a positive real number, we define

$$
G_{v, \alpha}\left(f_{1}, f_{2}, \ldots f_{N}\right)=\left(\left(\begin{array}{c}
N \\
v
\end{array}\right)^{-1} \sum f_{i_{1}}^{\alpha} f_{i_{2}}^{\alpha} \ldots f_{i_{v}}^{\alpha}\right)^{1 / \nu}
$$

for each $\left(f_{1}, f_{2}, \ldots, f_{N}\right) \in\left(C_{+}^{o}(X)\right)^{N}$, where the summation $\sum$ ranges over all the possible permutations $I_{v}=\left\{i_{1}, \ldots, i_{v}\right\}$ of $v$ integers contained in $\{1,2, \ldots, N\}$. It is evident that the composition map $U=G_{\nu, \alpha} \circ \xi^{N}$ is continuous and satisfies the strong Pareto condition and respect anonymity. As $(\nu, \alpha)$ has infinitely many combinations, the existence of infinitely many social utility maps as required is evident.

As we have explained in Sect. 2, the three results Theorems A, B and Corollary C are now proved. Theorem B is basically the extension of Jones-Zhang-Simpson's theorem on linear preferences to general ones. Their theorem states that there exist acceptable aggregation rules on linear preferences, if and only if null preference is allowed for the society but not for the individual. By "allowing null preferences", they do not mean the null preference is considered in the range space or in the domain. Rather, they mean that there is no open set containing null preference other than the entire space. It is equivalent to considering zero topology $\mathfrak{\Im}_{o}$ in our general setting. Theorem B shows its sufficiency, the substantial part of the theorem. The remained necessity follows directly from the nonexistence of a nontrivial continuous function

$$
F:\left(P, \Im_{o}\right)^{N} \longrightarrow\left(P, \Im_{o}\right) \text { or }(P, \Im)
$$

which can be proved easily. In fact, if such $F$ would exist, choose $q \in F\left(P^{N}\right)-F(\bar{o})$, where $\bar{o}=(o, o, \ldots, o)$, and $o \in P$ means the null preference, i.e., $\Lambda(o)=X$. Let 
$\bar{p}_{1}, \bar{p}_{2}, \ldots, \overline{p_{n}}, \ldots$ be a sequence in $P^{N}$ with $F\left(\bar{p}_{1}\right), F\left(\bar{p}_{2}\right), \ldots, F\left(\bar{p}_{n}\right), \ldots$ tending to $q \neq F(\bar{o})$ (we may take $\bar{p}_{1}=\bar{p}_{2}=\cdots=\bar{p}_{n}, \ldots=\bar{p}$, with $F(\bar{p})=q$ ). But $\Im_{o}$ topology yields that $\overline{p_{n}}$ tends to $\bar{o}$. This shows that $F$ is not continuous at $\bar{o}$, leading to a contradiction.

\section{Concluding remarks}

The notions of differentiable utility functions and topological preferences (i.e., continuous preference orders) are clear and well-defined. The former is an object of the differentiable structure of the continuum alternative space $X$ and it depends on the cardinality given by range $\boldsymbol{R}^{1}$. The latter is of topological structure, allowed to keep only ordinality. But there is no way to define consistently the notion of a "differentiable preference", which is an object of differentiable structure, while disregarding the cardinality of $\boldsymbol{R}^{1}$.

The previous approach from Antonelli to Chichilnisky to investigate preferences on a continuum alternative space $X$ is based on the notion of such a differentiable preference by constructing a differentiable unit vector field on $X$ with possible zeros somewhere. The construction would be successful if there were no zeros. The unit vectors imitate non-zero gradients of differentiable utility functions, by discarding their size. There inevitably happens an abrupt change when the gradients tend to zero, and presents inconsistency of the differentiable preference with the given differentiable structure. To fix the gap, it is necessary to have the framework to overlook the singular part, i.e., the part where the preference vectors are zero and therefore let preference convergence be rigid on singularity. That's why in topology $\mathfrak{s}_{c}$ of the differentiable framework, the separation property has to be imposed and the confusion arises, as illustrated in Sect. 1.1. An extreme case, though not often considered in social choice theory, illustrates the essence of the inconsistency of the differentiable framework. Namely, when $X=I^{1}$ or real line $R^{1}$, preference space $P_{c}$ is totally discrete in the sense that every preference is isolated from the rest of them and, consequently, there is no nontrivial convergence among preferences at all.

Normally, when topological behaviors are of main concern, such as when the existence problem of continuous rational social choice is investigated, a topological setting is the first priority to be considered. For example, the setting should start with continuous preference orders over $X$, which is natural and conventional. Why has it been replaced with the framework of differentiable settings as the main model? One reason is that the latter illustrates Condorcet paradox in a neat visible manner as Chichilnisky contributed (Chichilnisky (1982)). Another possible reason that I can think of is that a topological setting involves too many technical complications regarding mathematical treatment. A differentiable setting is relatively convenient to be employed. For instance, using the setting, an impossibility theorem with an elegant proof was immediately obtained by Chichilnisky. The technical complications have been avoided, but the confusion, as well as its implications, remained to be clarified.

We call the topological setting given in this paper "global", because the preferences under consideration are the continuous preference orders over the entire alternative 
space, in contrast to the "infinitesimal" change of differentiable utility functions that induces the notion of preference vector fields. Chichilnisky's differentiable setting is called "local" in this sense. As our topological setting is global, it has the advantage that the content of an inferior set $Q_{x}^{\prime}(p)$ can be measured quantitatively. This yields the existence of the required continuous social utility maps, and hence discloses the fact that the impossibility of having a continuous rational social welfare function is irrelevant to social aggregation itself.

The global topological setting may help clarify Chichilnisky's impossibility problem. Indeed, it also provides a rigorous foundation for the social choice theory on continuum alternative spaces.

As for the revised topology introduced by Jones, Zhang and Simpson (2003) following the line of the local differentiable setting to "allow" zero vectors in social preferences, we have constructed independently the zero order topology $\mathfrak{\Im}_{o}$, extending the revised topology on linear preferences, which they considered, to general ones in our global topological setting. We give a side evidence that a social aggregation apparatus such as a ballot counting machine can be arbitrarily accurate, so that it can detect arbitrarily slight difference of a social preference among alternatives, while individual preferences are relatively insensitive. This observation might justify the revised topology of Jones et al. (2003) and of our $\mathfrak{\Im}_{o}$ to be accepted for social preferences, and hence lead to the possibility results about continuous rational social welfare functions when the two topologies of individual and social preferences are not necessarily required to be the same (e.g., as stated in Theorem B). It was pointed out that the revised topology, or the zero order topology $\Im_{o}$, seems too weak to be a desirable topology for social preferences, e.g., a single sequence of social preferences may have two distinct limits. Nevertheless, if "God" arrange things in such an imperfect way, what could we do with it?

\section{Appendix}

(AIa) Proof of Proposition 2.3.1 (About how the topology of $X$ reflected on the index set $A)$ :

$\bigcap_{n=1}^{\infty} A_{\alpha_{n}} \supset A_{\alpha}$ is clear since $A_{\alpha_{n}} \supset A_{\alpha}, \forall n$. It suffices to show that $\bigcap_{n=1}^{\infty} A_{\alpha_{n}} \subset A_{\alpha}$. Suppose the contrary, i.e., $\exists \gamma \in \bigcap_{n=1}^{\infty} A_{\alpha_{n}}-A_{\alpha}$. Since $\gamma \in \bigcap_{n=1}^{\infty} A_{\alpha_{n}}$, we have $A_{\gamma} \subset$ $A_{\alpha_{n}}, \forall n$, by the minimality S4. Given $n$, we have Case 1: $\alpha_{n} \in A_{\gamma}$, which implies $A_{\alpha_{n}} \subset A_{\gamma}$, i.e., $A_{\alpha_{n}}=A_{\gamma}$. By the injectivity, $\alpha_{n}=\gamma$, and $I_{\alpha_{n}}=I_{\gamma}$. Case 2: $\alpha_{n} \notin A_{\gamma}$, which implies

$$
I_{\alpha_{n}} \subset X-\cup_{\beta \in A_{\gamma}} I_{\beta}
$$

In either case, we have $x_{n} \in Q_{\gamma}$, since

$$
x_{n} \in I_{\alpha_{n}} \subset\left(X-\cup_{\beta \in A_{\gamma}} I_{\beta}\right) \cup I_{\gamma} \equiv Q_{\gamma} .
$$


On the other hand, $\gamma \notin A_{\alpha}$ and $\gamma \in A_{\gamma}$. Hence, $A_{\gamma}$ is not contained in $A_{\alpha}$. By the linear ordering S3, $A_{\gamma} \supset A_{\alpha}$ with $\gamma \neq \alpha$. This together with the definition of $Q_{\gamma}$ yields that $I_{\alpha} \cap Q_{\gamma}=\phi$. But $x \in I_{\alpha}$; we have

$$
x \notin Q_{\gamma} .
$$

However, $x_{n} \in Q_{\gamma}$ and $x_{n} \rightarrow x$ in $X, \forall n$. But $Q_{\gamma}$ is closed in $X$ (due to S2), we would have $x \in Q_{\gamma}$. This is a contradiction.

(AIb) Proof of Proposition 2.3.2 (Equivalence between preferences and stratifications):

The proof is straightforward We show it here just for completion. Given $p \in P(X)$, we decompose $X$ into the indifference sets of $p$. The decomposition is well-defined by the transitivity of $\sim$ of $p$. Let the index set $A$ be the quotient set defined by $\sim$. We have $X=\cup_{\alpha \in A} I_{\alpha}$, where $I_{\alpha}=I_{x}(p), \forall x \in X$ with $x \in I_{\alpha}$. Hence S1 is satisfied. In the following, we simplify the notations by letting $I_{x} \equiv I_{x}(p), R_{x} \equiv R_{x}(p)$, $Q_{x} \equiv Q_{x}(p)$. Since $I_{\alpha}=I_{x}=R_{x} \cap Q_{x}$, each $I_{\alpha}$ is closed. For $x \in I_{\alpha}$, we define

$$
\left.A_{\alpha} \equiv\left\{\beta \in A ; \exists y \in I_{\beta} \text { with } y \succsim x \text { (in } p\right)\right\}
$$

then by the transitivity,we have $\beta \in A_{\alpha}$ iff $\forall z \in I_{\beta}, z \succsim x$. Hence,

$$
\begin{aligned}
R_{\alpha} & \equiv \cup_{\beta \in A_{\alpha}} I_{\beta}=\{y \in X ; y \succsim x\}=R_{x} \\
Q_{\alpha} & \equiv\left(X-\cup_{\beta \in A_{\alpha}} I_{\beta}\right) \cup I_{\alpha}=\{y \in X ; y \preceq x\}=Q_{x},
\end{aligned}
$$

and therefore $R_{\alpha}$ and $Q_{\alpha}$ are closed in $X$. This is S2. To show S3, let $I_{\alpha}=I_{x}, I_{\beta}=I_{y}$ with $x, y \in X$. Assume without loss of generality that $x \succsim y$. It holds that

$$
\begin{aligned}
A_{\alpha} \cap A_{\beta} & =\left\{\gamma \in A ; \forall z \in I_{\gamma}, z \succsim x \text { and } z \succsim y\right\} \\
& =\left\{\gamma \in A ; \forall z \in I_{\gamma}, z \succsim x\right\} \quad \text { (by transitivity of } p \text { ) } \\
& =A_{\alpha}
\end{aligned}
$$

As for S4, we have $\alpha \in A_{\alpha}, \forall \alpha \in A$, since if $I_{\alpha}=I_{x}$ for some $x$ then $y \succsim x$ $\forall y \in X_{\alpha}=I_{x}$. To claim that $A_{\beta} \subset A_{\alpha}, \forall \beta \in A_{\alpha}$, we let $\gamma \in A_{\beta}$. Let $I_{\alpha}=I_{x}$ and $y \in I_{\beta}$, we have $y \succsim x$. However, $\forall z \in X_{\gamma}$, we have $z \succsim y$. Hence $z \succsim x$ by the transitivity of $p$, and therefore $\gamma \in A_{\alpha}$.

Conversely, given $\sigma \equiv\left(X, I_{\alpha} ; A, A_{\alpha}\right)$ we define a preference $p$ on $X$ by

$$
x \precsim y \text { in } p \text { iff } A_{\alpha} \supset A_{\beta}
$$

where $x \in I_{\alpha}$ and $y \in I_{\beta}$. Evidently, $p$ is a preference order on $X$. In fact, by S3, we have $A_{\alpha} \supset A_{\beta}$ or $A_{\beta} \supset A_{\alpha}$ or both. The previous two cases correspond to $x \precsim y$ and $y \precsim x$ respectively. When $A_{\alpha}=A_{\beta}$, we have $\alpha=\beta$ by the injectivity. Hence $I_{\alpha}=I_{\beta}$ and $x, y \in I_{\alpha}$, i.e., $x \sim y$. So $p$ is complete. The transitivity of $p$ follows from the transitivity of set inclusion. Finally we have to show that $p$ respects the topology of 
$X$, in order that $p$ becomes a preference on $X$. By S3 and S4, it is clear that $\beta \in A_{\alpha}$ iff $A_{\alpha} \supset A_{\beta}$, and therefore iff $y \succsim x$. Hence

$$
\{y \in X ; y \succsim x\}=\cup_{\beta \in A_{\alpha}} I_{\beta},
$$

i.e., $R_{x}=R_{\alpha}$. Thus $R_{x}$ is closed in $X$, by S2. Similarly,

$$
\{y \in X ; y \precsim x\}=\left(X-\cup_{\beta \in A_{\alpha}} I_{\beta}\right) \cup I_{\alpha}
$$

i.e., $Q_{x}=Q_{\alpha}$. We also have $Q_{x}$ closed in $X$. By the following Proposition 2.2.1, the graph $G$ defining the preference order $p$ is closed in $X \times X$. The proof would then be completed.

(AII) Proof of Theorem 1 (Uniform approximation theorem)

Step 1 Let $W^{c} \equiv X-W$. Evidently, $W^{c}$ is also a compact set. Let

$$
L_{1} \equiv W^{c} \cap Q_{x}^{\prime}\left(p_{o}\right), L_{2} \equiv W^{c} \cap R_{x}^{\prime}\left(p_{o}\right)
$$

Then $L_{1} \cup L_{2}=W^{c}$. Clearly, $L_{1}$ and $L_{2}$ are disjoint and complement to each other. But they are open in $W^{c}$ and hence closed in $W^{c}$. As $X$ is compact, $L_{1}$ and $L_{2}$ are also compact. Also, $L_{1} \prec x \prec L_{2}$ in $p_{o}$. By the argument used in Step 1 of the proof of Proposition 2.2.1, $\exists x_{1} \in L_{1}$ and $x_{2} \in L_{2}$ with $L_{1} \precsim x_{1}$ and $x_{2} \precsim L_{2}$ in $p_{o}$. Let $V$

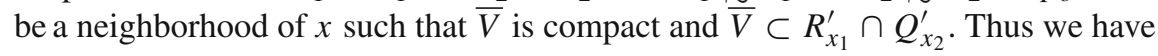

$$
L_{1} \precsim x_{1} \prec \bar{V} \prec x_{2} \precsim L_{2} \text { in } p_{o}
$$

Now $p_{o} \in \Gamma\left(L_{1}, \bar{V}\right) \cap \Gamma\left(\bar{V}, L_{2}\right)$. By the definition of $p_{n} \rightarrow p_{o}$ in $\Im$, there exists $N$ such that $p_{n} \in \Gamma\left(L_{1}, \bar{V}\right) \cap \Gamma\left(\bar{V}, L_{2}\right), \forall n>N$. This means that $\forall n>N$ and $\forall v \in V$,

$$
L_{1} \prec v \prec L_{2} \text { in } p_{n} \text {. }
$$

In particular, given $v \in V$, any point of $X$ indifferent to $v$ in $p_{n}$ with $n>N$ and $v \in V$ is disjoint from $W^{c} \equiv L_{1} \cup L_{2}$. It says that

$$
I_{v}\left(p_{n}\right) \subset W, \forall n>N \text { and } \forall v \in V .
$$

Step 2 When constructing the proof of the second statement, we keep in mind the fact mentioned in Example 24 where $K$ is not connected. Given a connected set $K$ with $x \in K \subset$ Int $I_{x}\left(p_{o}\right)$. Since $X$ is a locally connected, locally compact, $T_{3}$-space, $\exists V$ open in $X$ with its compact closure $\bar{V}$ connected such that

$$
x \in K \subset V \subset \bar{V} \subset \text { Int } I_{x}\left(p_{o}\right)
$$


Evidently, $\forall v \in V$,

$$
v \in \bar{V} \subset \operatorname{Int} I_{v}\left(p_{o}\right)
$$

We have $p_{o} \in \Omega(\bar{V}, X)$. By Definition 2.5.2, $\exists N_{1}$ such that $p_{n} \in \Omega(\bar{V}, X), \forall n>N_{1}$; i.e., $\bar{V} \subset \Lambda^{o}\left(p_{n}\right), \forall n>N_{1}$. For any fixed $n>N_{1}$ and each given $v \in V$ we claimed that

$$
\bar{V} \subset \operatorname{Int} I_{v}\left(p_{n}\right)
$$

Let $K_{o} \equiv \bar{V} \cap I_{v}\left(p_{n}\right)$. The indifference set $I_{v}\left(p_{n}\right)$ is closed in $X$. Clearly, $K_{o} \neq \phi$ since $v \in K_{O}$. Also, $K_{O}$ is closed in $\bar{V}$, We then claim that $K_{O}$ is also open in $\bar{V}$. It suffices to show that $K_{o} \subset$ Int $I_{v}\left(p_{n}\right)$. For any $y \in K_{o}$, we have $y \in \bar{V}$ and $y \sim v$ in $p_{n}$ due to the definition of $K_{o}$. Since $\bar{V} \subset \Lambda^{o}\left(p_{n}\right)$, it holds that $y \in \Lambda^{o}\left(p_{n}\right)$. By the definition of the interior singular set $\Lambda^{o}\left(p_{n}\right), \exists U$ open in $X$ with

$$
y \in U \subset I_{y}\left(p_{n}\right) \text {, i.e., } y \in \text { Int } I_{y}\left(p_{n}\right) \text {. }
$$

However, $I_{y}\left(p_{n}\right)=I_{v}\left(p_{n}\right)$. Therefore, $y \in \operatorname{Int} I_{v}\left(p_{n}\right)$ and hence

$$
K_{o} \subset \text { Int } I_{v}\left(p_{n}\right)
$$

In other words, $K_{o}=\bar{V} \cap \operatorname{Int} I_{v}\left(p_{n}\right)$. It follows that $K_{o}$ is open in $\bar{V}$. By the connectedness of $\bar{V}, \bar{V}$ contains no proper subset which is both open and closed. We have $\bar{V}=K_{o}$, and consequently,

$$
K \subset \bar{V} \subset \operatorname{Int} I_{v}\left(p_{n}\right),
$$

which completes the proof.

\section{(AIIa)Proof of Corollary 3.1.1}

The necessity has been proved in Sect. 2.5 and in Theorem 1. We show the sufficiency. Given $p_{n}, p_{o} \in P(X)$, satisfying (A1) and (A2), let $p_{o} \in \Gamma(K, L) \cap \Omega(J, W)$, we claim $\exists N_{o} \ni p_{n} \in \Gamma(K, L) \cap \Omega(J, W), \forall n>N_{o}$. The proof is straightforward.

Step 1 For $x \in K, y \in L$, we have $x \prec y$ in $p_{o}$. Note that $K$ and $L$ are compact. By transitivity, $I_{x} \prec I_{y}$ in $p_{o}$. Then (A1) implies $I_{x} \prec I_{y}$ in $p_{n}, \forall n>$ some $n_{o}$. Hence $x \prec y$ in $p_{n}, \forall n>n_{o}$. By Proposition 2.5.1 and applying the argument of finite open covering on $K$ and $L$, we have $p_{n} \in \Gamma(K, L), \forall n>n_{o}$.

Step 2 As $p_{o} \in \Omega(J, W)$, by definition of $\Omega, J$ is compact and non-empty, $W$ is open in $X$ and $J \subset \Lambda^{o}\left(p_{o}\right) \subset \Lambda\left(p_{o}\right) \subset W$. Let $\Lambda\left(p_{o}\right)=\stackrel{\infty}{\cup} I_{x_{i}}\left(p_{o}\right)$ and $\left\{x_{i_{1}}, \ldots, x_{i_{k}}, \ldots\right\}$ be a subsequence of $\left\{x_{i}\right\}$ such that $J_{i_{k}} \equiv I_{x_{i_{k}}}\left(p_{o}\right) \cap J \neq \phi$. Since $J_{i_{k}} \subset \Lambda^{o}\left(p_{o}\right), J_{i_{k}} \subset \operatorname{Int} I_{x_{i_{k}}}\left(p_{o}\right)$ and $J_{i_{k}}$ is open in $J$. Hence $\left\{J_{i_{k}}\right\}$ is an open covering of $J$. By $J$ compact, there are only a finite number $l$ of $J_{i_{k}}$. Denote $C_{k} \equiv J_{i_{k}}, k=1, \ldots, l$. For each $k$, consider

$$
C_{k} \subset \operatorname{Int} I_{x_{i_{k}}}\left(p_{o}\right) \subset I_{x_{i_{k}}}\left(p_{o}\right) \subset W .
$$


By(A2) (the precise definition is given in Theorem 1), $\exists N_{k}$ such that the last formula is valid if $p_{o}$ is replaced by $p_{n}, \forall n>N_{k}$. Let $\bar{N} \equiv \max \left\{N_{1}, \ldots, N_{l}\right\}$. As $J=\cup_{k=1}^{l} C_{k}$, we obtain $p_{n} \in \Omega(J, W), \forall n>\bar{N}$.

Step 3 Choose $N_{o}=\max \left\{n_{o}, \bar{N}\right\}$. Then the claim is proved. (AIIIa) Proof of Lemma A

(i) The proof of $\Lambda^{o}(p) \subset$ Int $\Lambda(p)$ is trivial. We claim the reverse. Let $y \in$ Int $\Lambda(p)$ in $X$.Then there exists a neighborhood $B$ of $y$ in $X$, such that $B \subset$ $\Lambda(p)$. We may let $B$ be an open ball, so that $B$ is connected, as $X=\mathbf{I}^{n}$. Since the topology of $X$ has a base of countably many open sets, $\Lambda^{o}(p)$ has at most countably many components, Thus $\Lambda(p)$ can be expressed by a countable union of $I_{x_{i}}(p)$ with each $x_{i}$ an interior singular point, i.e., $\Lambda(p)=\cup_{i=1}^{\infty} I_{x_{i}}(p)$, But each $I_{x_{i}}(p)$ is closed in $X$. It is observed that any two distinct $I_{x_{i}}(p)$ and $I_{x_{j}}(p)$ are disjoint (Suppose otherwise, $\exists z \in I_{x_{i}}(p) \cap I_{x_{j}}(p)$, then $\forall v \in I_{x_{i}}(p)$ and $w \in I_{x j}(p), v \sim z \sim w$, which implies $I_{x_{i}}(p)=I_{x_{j}}(p)$, contradiction). Denote $I_{i} \equiv I_{x_{i}}(p) \cap B$, then each $I_{i}$ is a closed subset of $B$ such that they are disjoint from each other. Now $B \subset \Lambda(p)$. It means that $B$ is a disjoint union of at most countably many closed sets $I_{i}^{\prime}$, which is possible only when there is exactly one non-empty $I_{i}$, i.e., $B=I_{i}$ (Even in the weird example 16, looking at $y \equiv \frac{1}{3}, B \equiv\left(\frac{1}{6}, \frac{1}{2}\right)$, there are countably many indifference sets $I_{x_{i}}(p)$ in $\Lambda(p)$ which accumulate around $y$, but $B$ is still a countable union of such corresponding $I_{i}^{\prime}$ s.) Remark that in the last statement we have used the fact that $B$ is not a union of countable disjoint and non-empty closed sets, which is equivalent to the theorem that Cantor's set in the unit interval has uncountable points in its complement. Therefore $y \in B=I_{i} \subset I_{x_{i}}(p)$ and $y$ is an interior point of $I_{x_{i}}(p)$. i.e., $y \in \Lambda^{o}(p)$.

(ii) That $\partial(\Lambda(p)) \supset \cup_{i=1}^{\infty} \partial\left(I_{x_{i}}(p)\right)$ follows directly from (i).

(iii) When $\Lambda(p)$ is assumed closed in $X$, we have $\partial(\Lambda(p)) \subset \Lambda(p)=\cup_{i=1}^{\infty} I_{x_{i}}(p)$. Given $y \in \partial(\Lambda(p))$, we have $y \in I_{x_{i}}(p)$ for some $i$. However, $y \notin \operatorname{Int}\left(I_{x_{i}}(p)\right)$, otherwise it violates $y \in \partial\left(\Lambda(p)\right.$. Then it is necessary that $y \in \partial\left(I_{x_{i}}(p)\right)$. Thus

$$
\partial\left(\Lambda(p) \subset \cup_{i=1}^{\infty} \partial\left(I_{x_{i}}(p)\right)\right.
$$

By (ii), we conclude that $\partial\left(\Lambda(p)=\cup_{i=1}^{\infty} \partial\left(I_{x_{i}}(p)\right)\right.$.

(AIIIb) Proof of Theorem 2 (The lifting theorem )

Step 1 Given $p \in P$, we first claim that the lifting function $f(x)$ is continuous in $x$. Let $\varepsilon>0$, choose a $\mu$-measurable compact set $L \subset Q_{x}^{\prime} \equiv Q_{x}^{\prime}(p)$ such that

$$
\mu\left(Q_{x}^{\prime}-L\right)<\frac{\varepsilon}{4}
$$

and choose an open set $W \supset Q_{x} \equiv Q_{x}(p)$ such that

$$
\mu\left(W-Q_{x}\right)<\frac{\varepsilon}{4}
$$


Let the notation "+" applied between two sets denotes the "disjoint union" of them, where the two sets have been assumed disjoint. Correspondingly, the summation notation $\sum$ stands for the union of a family of disjoint sets. Note that $Q_{x}=Q_{x}^{\prime}+I_{x}$. We have

$$
\begin{aligned}
\mu\left(W-I_{x}-L\right) & =\mu\left(\left(W-Q_{x}\right)+\left(Q_{x}-Q_{x}^{\prime}\right)+\left(Q_{x}^{\prime}-L\right)-I_{x}\right) \\
& =\mu\left(\left(W-Q_{x}\right)+\left(Q_{x}^{\prime}-L\right)\right)<\frac{\varepsilon}{4}+\frac{\varepsilon}{4}=\frac{\varepsilon}{2}
\end{aligned}
$$

Since $Q_{x}-Q_{x}^{\prime}=I_{x}$. Clearly, $x \in W-L$, and $W-L$ is open in $X$. Based on Proposition 2.2.2, we may choose $V$, open in $X$, with $x \in V \subset$ the compact closure $\bar{V} \subset W-L$, such that $L \prec \bar{V} \prec X-W$. Then $\forall y \in \bar{V}$, we have

$$
L \subset Q_{y}^{\prime} \equiv Q_{y}^{\prime}(p) \subset W
$$

It suffices to show that

$$
|f(y)-f(x)|<\varepsilon, \quad \forall y \in \bar{V} .
$$

Let $\Lambda$ denotes $\Lambda(p)$ for abbreviation. Decompose $\Lambda=\Lambda^{\prime}+\Lambda^{\prime \prime}$, where $\Lambda^{\prime} \subset L$ and $\Lambda^{\prime \prime} \subset X-L$. We have

$$
\begin{aligned}
f(y) & =\mu\left(Q_{y}^{\prime}-\Lambda\right)+1=\mu\left(Q_{y}^{\prime}-\Lambda^{\prime \prime}-\Lambda^{\prime}\right)+1 \\
& =\mu\left(\left(Q_{y}^{\prime}-\Lambda^{\prime \prime}-L\right)+\left(L-\Lambda^{\prime}\right)\right)+1 \\
& =\mu\left(Q_{y}^{\prime}-L-\Lambda^{\prime \prime}\right)+\mu\left(L-\Lambda^{\prime}\right)+1
\end{aligned}
$$

But by (2), $Q_{y}^{\prime}-L-\Lambda^{\prime \prime} \subset W-L-\Lambda^{\prime \prime} \subset W-L-I_{x}$. Hence by (1),

$$
|f(y)-f(x)| \leq 2 \mu\left(W-L-I_{x}\right)<\varepsilon
$$

which proves (3). Hence $f(x)$ is continuous in $x$.

Step 2 Consider $\mathcal{F}(K, \widetilde{U})$ where $K$ is a compact set of $X$ and $\widetilde{U}$ is open in the real line $\mathbf{R}^{1}$. In order to show the continuity of $\xi$, it suffices to show that $\xi^{-1}(\mathcal{F}(K, \widetilde{U}))$ is open in $P$, . Let $p \in \xi^{-1}(\mathcal{F}(K, \widetilde{U}))$, i.e., the function $f \equiv \xi(p)$ maps $K$ into $\widetilde{U}$.

Given $x \in K$ with $f(x) \in \widetilde{U}$. Choose $\varepsilon>0$, such that the interval $(f(x)-\varepsilon$, $f(x)+\varepsilon) \in \widetilde{U}$. We first claim that there exist a compact neighborhood $V$ of $x$ in $X$ and a neighborhood $S$ of $p$ in $P$ such that

$$
\xi(q) \text { maps } V \text { into } \widetilde{U}, \forall q \in S
$$

Step 3 (i) Choose $L_{1}$ compact in $Q_{x}^{\prime}(p)$ such that

$$
\mu\left(Q_{x}^{\prime}(p)-L_{1}\right)<\frac{\varepsilon}{4}
$$


Let $y \in L_{1}$, we have $y \prec x$ in $p$. By Proposition 2.2.2, $\exists U_{y^{\prime}}^{\prime} V_{x}^{\prime}$, compact neighborhoods of $y, x$, respectively such that

$$
U_{y}^{\prime} \prec V_{x}^{\prime} \text { in } p
$$

Hence $p \in \Gamma\left(U_{y}^{\prime}, V_{x}^{\prime}\right)$. Cover $L_{1}$ by the union of all such $U_{y}^{\prime}$, with $y \in L_{1}$, Since $L_{1}$ is compact, $\exists$ finite subcover

$$
\left\{U_{y_{1}}^{\prime}, \ldots, U_{y_{k}}^{\prime}\right\}
$$

of $L_{1}$. Consider the corresponding $\left\{V_{1}^{\prime}, \ldots, V_{k}^{\prime}\right\}$ with $x \in V_{i}^{\prime}$ and $U_{y_{i}}^{\prime} \prec V_{i}^{\prime}, \forall i$. Denote

$$
S_{1} \equiv \cap_{i=1}^{h} \Gamma\left(U_{y_{i}}^{\prime}, V_{i}^{\prime}\right), V_{1} \equiv \cap_{i=1}^{h} V_{i}^{\prime}
$$

Then $p \in S_{1}$ and the set $S_{1}$ is open in $P$. Thus $\forall q \in S_{1}$ and $v \in V_{1}$, we have

$$
L_{1} \subset Q_{v}^{\prime}(q)
$$

(In this sense we may say that $Q_{x}^{\prime}(p)$ is "lower semi-continuous in $p$ ", as $L_{1}$ is arbitrarily close to $Q_{x}^{\prime}(p)$ from the interior. See also (IV) of Introduction.)

(ii) Similarly, choose $L_{2}$ compact in $R_{x}^{\prime}(p)$, such that

$$
\mu\left(R_{x}^{\prime}(p)-L_{2}\right)<\frac{\varepsilon}{4}
$$

Let $W \equiv X-L_{2}$. We now claim that there exist a compact neighborhood $V_{2}$ of $x$ in $X$ and a neighborhood $S_{2}$ of $p$ in $P$ such that $V_{2} \subset W$ and

$$
Q_{v}^{\prime}(q) \subset W, \forall q \in S_{2} \text { and } v \in V_{2}
$$

By the argument given in (i), we can have

$$
L_{2} \subset R_{v}^{\prime}(q), \forall q \in S_{2} \text { and } v \in V_{2}
$$

which implies that

$$
Q_{v}^{\prime}(q) \subset Q_{v}(q)=X-R_{v}^{\prime}(q) \subset X-L_{2}=W,
$$

$\forall q \in S_{2}$ and $v \in V_{2}$.

Step 4 Since each singular indifference set $I_{x_{i}}(p)$ is $\mu$-measurable, $\Lambda(p)=\cup_{i=1}^{\infty}$ $I_{x_{i}}(p)$ is $\mu$-measurable. There exist two unions $E$ and $F$ of finitely many closed $n$-rectangles such that

$$
E \subset \Lambda(p) \subset \text { Int } F \subset F \text { and } \mu(F-E)<\frac{\varepsilon}{8}
$$


(Note that this is not true in Example 17a, where $\Lambda(p)$ is not $\mu$-measurable). But now Int $E \subset$ Int $\Lambda(p)=\Lambda^{o}(p)$ where the last equality is established by Lemma A.

Step 5 Choose a compact set $L_{3} \subset$ Int $E$ such that

$$
\mu\left(E-L_{3}\right)=\mu\left(\text { Int } E-L_{3}\right)<\frac{\varepsilon}{8}
$$

noting that $\mu(E-$ Int $E)=0$. We see that

$$
S_{3} \equiv \Omega\left(L_{3}, \text { Int } F\right)
$$

is an open neighborhood of $p$, where $\forall q \in S_{3}$.

$$
L_{3} \subset \Lambda^{o}(q) \subset \Lambda(q) \subset \text { Int } F \equiv F_{o}
$$

By (9) and (10), we have

$$
\mu\left(F_{o}-L_{3}\right)<\frac{\varepsilon}{8}+\frac{\varepsilon}{8}=\frac{\varepsilon}{4}
$$

Step 6 By (6), (8) and (11), we have

$$
L_{1}-F_{o} \subset Q_{v}^{\prime}(q)-\Lambda(q) \subset W-L_{3}
$$

and hence the difference

$$
\begin{aligned}
& \left|\mu\left(Q_{v}^{\prime}(q)-\Lambda(q)\right)-\mu\left(Q_{x}^{\prime}(p)-\Lambda(p)\right)\right| \\
& \quad \leq \mu\left(\left(W-L_{3}\right)-\left(L_{1}-F_{o}\right)\right),
\end{aligned}
$$

$\forall q \in S \equiv S_{1} \cap S_{2} \cap S_{3}$ and $\forall v \in V \equiv V_{1} \cap V_{2}$, since by (6) and (11)

$$
L_{1}-F_{o} \subset Q_{x}^{\prime}(p)-\Lambda(p)
$$

But

$$
\begin{aligned}
\mu\left(\left(W-L_{3}\right)-\left(L_{1}-F_{o}\right)\right) & \leq \mu\left(\left(W-L_{3}\right)-\left(L_{1}-L_{3}\right)\right)+\mu\left(F_{o}-L_{3}\right) \\
& \leq \mu\left(W-L_{3}-L_{1}\right)+\frac{\varepsilon}{4} \quad(\text { by }(12)) \\
& \leq \mu\left(W-\left(L_{3} \cap I_{x}(p)\right)-L_{1}\right)+\frac{\varepsilon}{4} \\
& \leq \mu\left(\left(X-L_{2}\right)-\left(L_{3} \cap I_{x}(p)\right)-L_{1}\right)+\frac{\varepsilon}{4} \\
& \leq \mu\left(\left(R_{x}^{\prime}(p)-L_{2}\right) \cup\left(I_{x}(p)-L_{3}\right) \cup\left(Q_{x}^{\prime}(p)-L_{1}\right)\right)+\frac{\varepsilon}{4} \\
& \leq\left(\frac{\varepsilon}{4}+\frac{\varepsilon}{4}+\frac{\varepsilon}{4}\right)+\frac{\varepsilon}{4}=\varepsilon \quad(\text { by }(7),(12),(5))
\end{aligned}
$$


It shows that

$$
|\xi(q)(v)-\xi(p)(x)|<\varepsilon
$$

In other words, $\forall q \in S$,

$$
\xi(q) \text { maps } V \text { into } \widetilde{U}
$$

Thus the claim (4) of step 2 is proved.

Step 7 By Step 6, there exists for each $x \in K$ a neighborhood $V_{x}$ of $x$ in $X$ and a neighborhood $S_{x}$ of $p$ in $P$ such that

$$
\xi(q) \text { maps } V_{x} \text { into } \widetilde{U}, \forall q \in S_{x} .
$$

By $K$ compact, there exists a finite cover $\left\{V_{x_{1}}, V_{x_{2}}, \ldots, V_{x_{h}}\right\}$ of $K$. Choose

$$
S_{o} \equiv S_{x_{1}} \cap S_{x_{2}} \cap \cdots \cap S_{x_{h}}
$$

We have $\forall q \in S_{o}$,

$$
\xi(q) \text { maps } K \text { into } \widetilde{U}
$$

i.e., $\xi(q) \in \mathcal{F}(K, \widetilde{U})$. In other words, the open neighborhood $S_{o}$ of $p$ lies in $\xi^{-1}(\mathcal{F}(K, \widetilde{U}))$, for any given $p \in \xi^{-1}(\mathcal{F}(K, \widetilde{U}))$. It shows that $\xi^{-1}(\mathcal{F}(K, \widetilde{U}))$ is open in $P$. The proof of the continuity of $\xi$ is completed.

(AIVa) Let $X=[-1,1] \times[-1,1] \subset \mathbf{R}^{2}$, and $a_{n} \in(1 / n, 0) \in X$. Define preference vector fields $p_{n}=(0,0)$ at $a_{n}$ and $(0,1)$ elsewhere. Define $p=(0,0)$ at $a_{n}$, for any $n$, and $(0,1)$ elsewhere. Then singular set $S_{n}$ of $p_{n}$ is $a_{n}$ and that of $p$ is $\cup S_{n}=\left\{a_{1}, a_{2}, \ldots, a_{n}, \ldots\right\}$. Note that $\left|p_{n}(x)-p(x)\right|=0, \forall x \in X-\cup S_{n}$. According to the formula (**) in Remark A of Sect. 1.1, which was defined by Chichilnisky (Chichilnisky 1982, pp349), it is clear that $\left\{p_{1}, p_{2}, p_{3}, \ldots, p_{n}, \ldots\right\}$ converges to $p$. But $\left\{p_{2}, p_{4}, p_{6}, \ldots, p_{2 k}, \ldots\right\}$ does not converge to $p$. In fact,

$$
X-\cup_{k=1}^{\infty} S_{2 k}=\left\{a_{1}, a_{3}, a_{5} \ldots\right\}
$$

and $\left|p_{2 k}\left(a_{1}\right)-p\left(a_{1}\right)\right|=|(0,1)-(0,0)|=1$, so the sup norm is 1 . Furthermore, a similar argument shows that even sequence $\left\{p_{2}, p_{3}, p_{4}, p_{5}, \ldots, p_{n}, \ldots\right\}$ does not converge to $p$. This is weird. It says that the formula $(* *)$ does not define a topology, nor the notion of continuity.

(AIVb) We will show that $d_{c}^{*}(p, q)$ given by a symmetric form, $\sup _{x \in X-S_{p} \cup S_{q}}$ | $p(x)-q(x) \mid$, is also not a distance:

(1) It is not positive definite, since we may construct an example with $d_{c}^{*}(p, q)=0$ does not imply $p=q$. In fact, let $A \varsubsetneqq B \varsubsetneqq X=I$ and $p=(0,0)$ on $A$, yet $=(0,1)$ elsewhere. Let $q=(0,0)$ on $B$, yet $=(0,1)$ elsewhere. It yields that singular sets $S_{p}=A \varsubsetneqq B=S_{q}$. Clearly, $p=(0,1)=q$ on $X-S_{p} \cup S_{q}$. So $d_{c}^{*}(p, q)=0$, but $p \neq q$. 
(2) The triangular inequality is not satisfied: Let $X=\{(x, y) ; 0 \leq x \leq 1,0 \leq$ $y \leq 1\}$. Let $e_{1}=(1,0)$ and $e_{2}=(0,1)$ be the canonical orthonormal frame of $R^{2}$.Consider three preference vector fields $p, q$, and $r$ as follows. Let

$$
\begin{aligned}
& p=\sin \pi y e_{1}+\cos \pi y e_{2} \\
& q=\sin \pi y e_{1}-\cos \pi y e_{2}
\end{aligned}
$$

for $0 \leq y \leq 1 / 2$, i.e., as $y$ changes from 0 to $1 / 2, p$ turns clockwise from $e_{2}$ to $e_{1}$ for 90 degrees, and yet $q$ turns counter clockwise. Furthermore, let $r=(0,0)$ for $0 \leq y \leq 1 / 2$, and $p=q=r=e_{1}$ for $1 / 2 \leq y \leq 1$. Then $d_{c}^{*}(p, q)=2>d_{c}^{*}(p, r)+d_{c}^{*}(r, q)=0$, where $d_{c}^{*}(p, r)=d_{c}^{*}(r, q)=0$, since $S_{r}=\{0 \leq y \leq 1 / 2\}, S_{p}=S_{q}=$ empty set $\phi$, and $X-S_{p} \cup S_{r}=X-S_{r} \cup S_{q}=$ $\{1 / 2 \leq y \leq 1\}$ on which $p=q=r$.

(AIVc) If the preference space of form $\gamma$ (i.e., it is the quotient topological space $F / \sim$ of function space $F$ on $X$ ), or of form $\delta$, is considered, Chichilnisky's proof of her theorem is invalid: Looking at Sect. 1.1, the range space $S^{1} \cup\{0\}$ of induced map $F^{\prime \prime}: S^{1} \times S^{1} \rightarrow S^{1} \cup\{0\}$ is now a connected set. It is even not a $T_{1}$-space, i.e., not every point is a close point. In fact, given an equivalent class [ $f]$ with $f \in F$, we have $f \sim t f$ for any $t>0$, and therefore $[f] \sim[t f]$. But $t f$ converges to zero function 0 , as $t \rightarrow 0$. By the quotient topology $\Im_{c}^{*}$ on $F / \sim,[t f]$ converges to the null preference $o=[0]$. Therefore, $\exists$ no open set in the preference space, which contains $o$ and is disjoint from $[f]=[t f]$. Hence $[f]$ is not a closed point, and $S^{1} \cup\{0\}$ in this topology $\mathfrak{\Im}_{c}^{*}$ becomes a connected set. This makes the proof of impossibility theorem invalid. The argument about the preference space of form $\delta$ is the same. It is noteworthy to remark that the topology $\Im_{c}^{*}$ of form $\gamma$ is equivalent to $\Im_{o}$ defined in Definition 2.5.1. The equivalence is not difficult to prove (see Huang 1996).

\section{References}

Arrow K (1951) Social choice and individual values. Wiley, New York

Baigent N (1987) Preference proximity and anonymous social choice. Quart J Econ 102:161-169

Chichilnisky G (1980) Social choice and the topology of spaces of preferences. Adv Math 37:165-176

Chichilnisky G (1982) Social aggregation rules and continuity. Quart J Econ 97(2):337-352

Chen HJ, Huang WH (2008) Existence of continuous rational social choices on infinite discrete alternatives (to appear)

Debreu G (1972) Smooth preferences. Econometrica 23:603-615

Huang WH (1996) Singularity in social choice theory, Technical Report. National Taiwan University, pp $1-64$

Huang WH (2004) Is proximity preservation rational in social choice theory? Soc Choice Welf 23:315-332 Jones M, Zhang J, Simpson G (2003) Aggregation of utility and social choice: a topological characterization. J Math Psychol 47:545-556

Kelly J (1955) General topology. Van Nostrand, New York

Mas-Collel A, Whiston W, Green J (1995) Microeconomic theory. Oxford Univ. Press, Oxford

Sen A (1995) Rationality and social choice. Am Econ Rev 85(1):1-24 